\title{
Article
}

\section{COVID-19 in People Living with HIV: A Systematic Review and Meta-Analysis}

\author{
Kai Wei Lee ${ }^{1,2}$, Sook Fan Yap ${ }^{1,2, *}$, Yun Fong Ngeow ${ }^{1,2}$ and Munn Sann Lye ${ }^{3}$ \\ 1 Department of Pre-Clinical Sciences, Faculty of Medicine and Health Sciences, Universiti Tunku Abdul \\ Rahman, Kajang 43000, Malaysia; lee_kai_wei@yahoo.com (K.W.L.); ngeowyf@utar.edu.my (Y.F.N.) \\ 2 Centre for Research on Communicable Diseases, Universiti Tunku Abdul Rahman, Kajang 43000, Malaysia \\ 3 Department of Population Medicine, Faculty of Medicine and Health Sciences, Universiti Tunku Abdul \\ Rahman, Kajang 43000, Malaysia; lyems9@yahoo.com \\ * Correspondence: yapsf@utar.edu.my
}

check for updates

Citation: Lee, K.W.; Yap, S.F.; Ngeow, Y.F.; Lye, M.S. COVID-19 in People Living with HIV: A Systematic Review and Meta-Analysis. Int. J. Environ. Res. Public Health 2021, 18, 3554. https://doi.org/10.3390/ ijerph18073554

Academic Editor: Paul B. Tchounwou

Received: 25 February 2021

Accepted: 15 March 2021

Published: 30 March 2021

Publisher's Note: MDPI stays neutral with regard to jurisdictional claims in published maps and institutional affiliations.

Copyright: (c) 2021 by the authors. Licensee MDPI, Basel, Switzerland. This article is an open access article distributed under the terms and conditions of the Creative Commons Attribution (CC BY) license (https:// creativecommons.org/licenses/by/ $4.0 /)$.

\begin{abstract}
COVID-19 is a global health emergency. People living with human immunodeficiency virus (PLHIV) have concerns about whether they have a higher risk of getting the infection and suffer worse COVID-19 outcomes. Findings from studies on these questions have largely been inconsistent. We aimed to determine the epidemiological characteristics, clinical signs and symptoms, blood parameters, and clinical outcomes among PLHIV who contracted COVID-19. Relevant studies were identified through Medline, Cinahl, and PubMed databases. A random-effects model was used in meta-analyses with a $95 \%$ confidence interval. Eighty-two studies were included in the systematic review and sixty-seven studies for the meta-analysis. The pooled incidence proportion of COVID-19 among PLHIV was $0.9 \%$ (95\% CI 0.6\%, 1.1\%) based on the data from seven cohort studies. Overall, 28.4\% were hospitalised, of whom, 2.5\% was severe-critical cases and 3.5\% needed intensive care. The overall mortality rate was $5.3 \%$. Hypertension was the most commonly reported comorbidity $(24.0 \%)$. Fever $(71.1 \%)$ was the most common symptom. Chest imaging demonstrated a wide range of abnormal findings encompassing common changes such as ground glass opacities and consolidation as well as a spectrum of less common abnormalities. Laboratory testing of inflammation markers showed that C-reactive protein, ferritin, and interleukin- 6 were frequently elevated, albeit to different extents. Clinical features as well as the results of chest imaging and laboratory testing were similar in highly active antiretroviral therapy (HAART)-treated and nontreated patients. PLHIV were not found to be at higher risk for adverse outcomes of COVID-19. Hence, in COVID-19 management, it appears that they can be treated the same way as HIV negative individuals. Nevertheless, as the pandemic situation is rapidly evolving, more evidence may be needed to arrive at definitive recommendations.
\end{abstract}

Keywords: COVID-19; HIV; AIDS; systematic review; meta-analysis

\section{Introduction}

The COVID-19 outbreak has been declared as a global health emergency by the World Health Organization [1]. Till date, the number of new COVID-19 cases has continued to increase daily in the backdrop of a lack of curative agents against the SARS-COV-2-virus. Vaccines have just been introduced but widespread implementation remains a significant issue. The clinical spectrum of COVID-19 ranges from mild through moderate to severe illness. Most infected individuals are asymptomatic or have a mild flu-like illness. Moderate and severe illness present with features of lower respiratory tract involvement that can proceed to respiratory failure, shock, and multiple organ failure, which can be fatal [2]. Common laboratory abnormalities of the infection are markers of systemic inflammation and coagulopathies, reflections of the underlying pathologies and complications of serious illness [2-4]. 
It is widely known and reported that older people, people who are immunocompromised, and those with underlying co-morbidities such as hypertension, diabetes mellitus, and pre-existing respiratory and cardiovascular disorders are at significantly higher risk of severe illness that requires hospitalization and intensive supportive therapy and care $[5,6]$. For example, people with hypertension had a significantly higher mortality risk from COVID-19 (pooled odds ratio $=3.36$ ) compared with the normotensive population [7]. A meta-analysis reported that the odds ratio for mortality among those with diabetes was 1.75 $(p<0.01)$ [8]. Patients with chronic obstructive pulmonary disease had higher risk for severe COVID-19 (pooled relative risk $=1.88, p<0.05$ ) [9]; likewise those with cardiovascular disease (odds ratio $=4.85, p<0.05$ ) [10] compared to those without these comorbidities.

A systematic review reported that among people living with human immunodeficiency (PLHIV) who contracted COVID-19, the most common co-morbidities were hypertension (39.3\%) and obesity (19.3\%) [11]. More than half (66.5\%) had mild-moderate symptoms, $74 \%$ had fever, and $58.3 \%$ had cough [11]. Another review indicated that PLHIV with well-controlled disease were not at higher risk for poorer COVID-19 outcomes than the general population [12]. However, these findings cannot be extrapolated to all PLHIV as these reviews are limited by small sample size and the disproportionate number of symptomatic COVID-19 cases who required hospitalization compared to those with mild or no symptoms of COVID-19. Further, the pooled incidence of COVID-19 and disease manifestations among PLHIV remains unknown. PLHIV often have concerns and questions related to their risk of serious illness from COVID-19 [13]. The limited availability of data emphasises the need for more studies to address these concerns. To date there is no specific treatment for COVID-19; patient management is largely supportive and symptomatic including the use of drugs to mitigate the complications of the infection [14,15].

In this rapidly evolving situation, clinicians and scientists are still learning about COVID-19 and how it affects PLHIV. Although the clinical characteristics of COVID-19 have been broadly described [16], there is still scarce literature on the clinical features including comorbidities, presenting symptoms and signs, severity, complications, and outcomes among PLHIV who contracted the infection. There are also uncertainties regarding the laboratory parameters that are predictive of disease severity and outcome of COVID-19 among PLHIV. Likewise, it is unclear whether factors related to the HIV infection and its management as well as patient compliance influence the clinical presentation, severity, and outcome of COVID-19. To date, the information reported in the literature has been inconsistent. We therefore conducted a systematic review and meta-analysis to determine the incidence proportion of COVID-19 among PLHIV and review the clinical course and outcomes in PLHIV co-infected with COVID-19. In addition, we also compared the clinical and laboratory features, and disease outcome between PLHIV who are on highly active antiretroviral therapy (HAART) and those who are not.

\section{Materials and Methods}

We conducted this review according to Preferred Reporting Items for Systematic Reviews and Meta-Analyses (PRISMA) (Supplementary Table S1) [17]. This study has been registered with PROSPERO (registration number: CRD42020210161).

\subsection{Literature Search}

Three databases (Medline, Cinahl, and PubMed) were searched on 20 September 2020 by two investigators independently (KWL and SFY) to identify potential studies. The search strategy consisted of several terms which were: (HIV OR human immunodeficiency virus* OR AIDS OR acquired immunodeficiency syndrome) AND (COVID-19 OR coronavirus OR 2019-NCOV OR nCoV* OR COVID* OR SARS-CoV*) with limiters of ENGLISH and HUMAN (Supplementary Table S2). We performed reverse and forward citation tracking; the last search was done on 26 October 2020. Papers published beyond this date were not considered for inclusion into this review. 


\subsection{Data Handling}

All relevant articles identified through the above databases were imported into the Endnote ${ }^{\circledR}$ programme version $X 5$, after which de-duplication was performed. Subsequently, titles and abstracts were reviewed for their relevance by two clinical pathologists (SFY and YFN) and the full texts of the selected articles were assessed for their eligibility to be recruited into this systematic review and meta-analysis.

\subsection{Selection Criteria}

Prospective and retrospective cohort studies, cross-sectional studies, case-control studies, case series, and case reports were eligible for inclusion. Studies were excluded if they were randomized controlled trials, controlled clinical trials or any type of review and meta-analysis. The studies must have data on the number of COVID-19 cases among HIV / AIDS patients or possess any data related to comorbidities, HIV profile, pharmacological treatment and supportive care for COVID-19, and laboratory test results. In addition, presenting symptoms and signs, imaging findings, and contact history were used as supplemental information for the evaluation of the diagnoses. We also examined the clinical outcomes of COVID-19, and the cause of death among those with HIV/AIDS infected with COVID-19. Any disagreements among the investigators were resolved through discussions and consultations with the third senior investigator (MSL) before the final consensus for quantitative analysis was reached.

\subsection{PICO}

The participants should be HIV patients (age $>18$ years) with a confirmed diagnosis of COVID-19 by nucleic-acid based testing, or with positive serological test and/or presence of typical radiological findings and clinical features of COVID-19. Exposure was referred to as COVID-19 infection. There was no comparator in the current review. The main outcomes for this review were pooled percentage (or pooled incidence proportion) of COVID-19 among those with HIV infection and the mortality rate due to COVID-19 among HIV-infected people. Secondary outcome was pooled prevalence of symptoms among HIV patients diagnosed with COVID-19.

\subsection{Data Extraction}

This step was performed independently by two investigators (KWL and SFY) and proofread by another two investigators (YFN and MSL). The information we extracted from the included studies were basic characteristics of studies as well as demographic characteristics, severity of disease, and mortality rate among patients with HIV-COVID-19 co-infection; data on comorbidities and lifestyle-related disorders, HIV profiles, diagnostic methods for COVID-19, vital signs, findings of chest imaging, pharmacological and supportive care given to treat the disease, and symptoms experienced by HIV-COVID-19 patients throughout the clinical course as well as the results of laboratory investigations (inflammation markers, liver profile, serum creatinine, serum lactate dehydrogenase, D-dimer, and full blood counts).

\subsection{Quality Assessment}

The quality of the papers included was independently assessed by two investigators (KWL and SFY). We used the checklist "Strengthening the Reporting of Observational Studies in Epidemiology" (STROBE) to assess the quality of cohort studies [18]. We used the quality appraisal checklist developed by the Institute of Health Economics to appraise individual studies pertaining to case series or case reports (Supplementary Table S3) [19].

\subsection{Operational Definition}

Advanced HIV / AIDS infection for adults is defined as those with clinical stage 3 or stage 4 disease or where CD4 count is available, with CD4 $<350$ cells $/ \mathrm{mm}^{3}$ at any clinical stage [20]. Undetectable viral load is defined as $<20 \mathrm{RNA}$ copies $/ \mathrm{mL}$. We used severity of 
COVID-19 as defined by the World Health Organization [21] (Supplementary Table S4). In brief, mild disease is defined as symptomatic disease meeting the case definition for COVID-19 with no evidence of viral pneumonia or hypoxia. Moderate disease is defined as symptomatic disease with clinical signs of pneumonia (such as fever, cough, dyspnoea, and increased rate of breathing) but without features of severe pneumonia. Severe disease is defined by clinical signs of pneumonia that are accompanied by a respiratory rate $>30$ breaths / min, or oxygen saturation less than $90 \%\left(\mathrm{SpO}_{2}<90\right)$ while breathing room air, or clinical symptoms of severe respiratory distress. Critical disease is defined as a disease that is complicated by either acute respiratory distress syndrome or sepsis/septic shock.

\subsection{Data Syntheses}

For meta-analysis, the incidence proportion of COVID-19 among PLHIV was derived from cohort studies that had inception cohorts of HIV patients without COVID-19 at baseline. For symptoms presented by patients with HIV-COVID-19 co-infection, data were derived from all cohort studies and case series with sample size not less than 30 people for meta-analysis. The results were pooled only if comparable data were available from more than two studies. Meta-analysis was performed with Open Meta (Analyst) ${ }^{\circledR}$ [22] using a random-effect model (DerSimonian and Laird method) to produce the pooled incidence proportion (PIP) or prevalence and their respective 95\% CIs. Heterogeneity was assessed using $\mathrm{I}^{2}$, with a $p$-value of less than 0.05 as significant. A sensitivity analysis was conducted using leave-one-out meta-analysis to examine how the exclusion of each individual study affects the overall estimate of the rest of the studies.

\section{Results}

\subsection{Search Results}

We identified 677 articles in the initial screening, as shown in Figure 1. After the removal of duplicate articles $(n=272)$, a total of 405 articles were retrieved for further assessment. After screening for suitability through the title and abstract, 107 articles were selected for full-text assessment. After careful evaluation, 82 articles were finally included for the systematic review, out of which, 7 cohort studies were deemed eligible for meta-analysis for PIP of COVID-19 among PLHIV and 26 studies (24 cohort studies and 2 case series) for pooled prevalence of symptoms. Only three studies [23-25] were used simultaneously for the systematic review and meta-analysis for PIP and pooled prevalence of symptoms.

\subsection{Description of Included Studies}

The main characteristics of the 82 studies are summarized in Table 1. A total sample of 643,018 HIV patients from 19 countries was included in the analysis. Twenty-four of these studies were in the USA, 14 in China, 12 in Italy, 5 in Spain and the UK, 4 in South Africa, and 3 in Japan. Of the remaining, two each were in Brazil, France, and Germany, and one each from Cyprus, India, Libya, Panama, Peru, Singapore, South Korea, Turkey, and Uganda. Out of 82 studies, 45 were case series, 26 studies were case reports and 11 were cohort studies. 


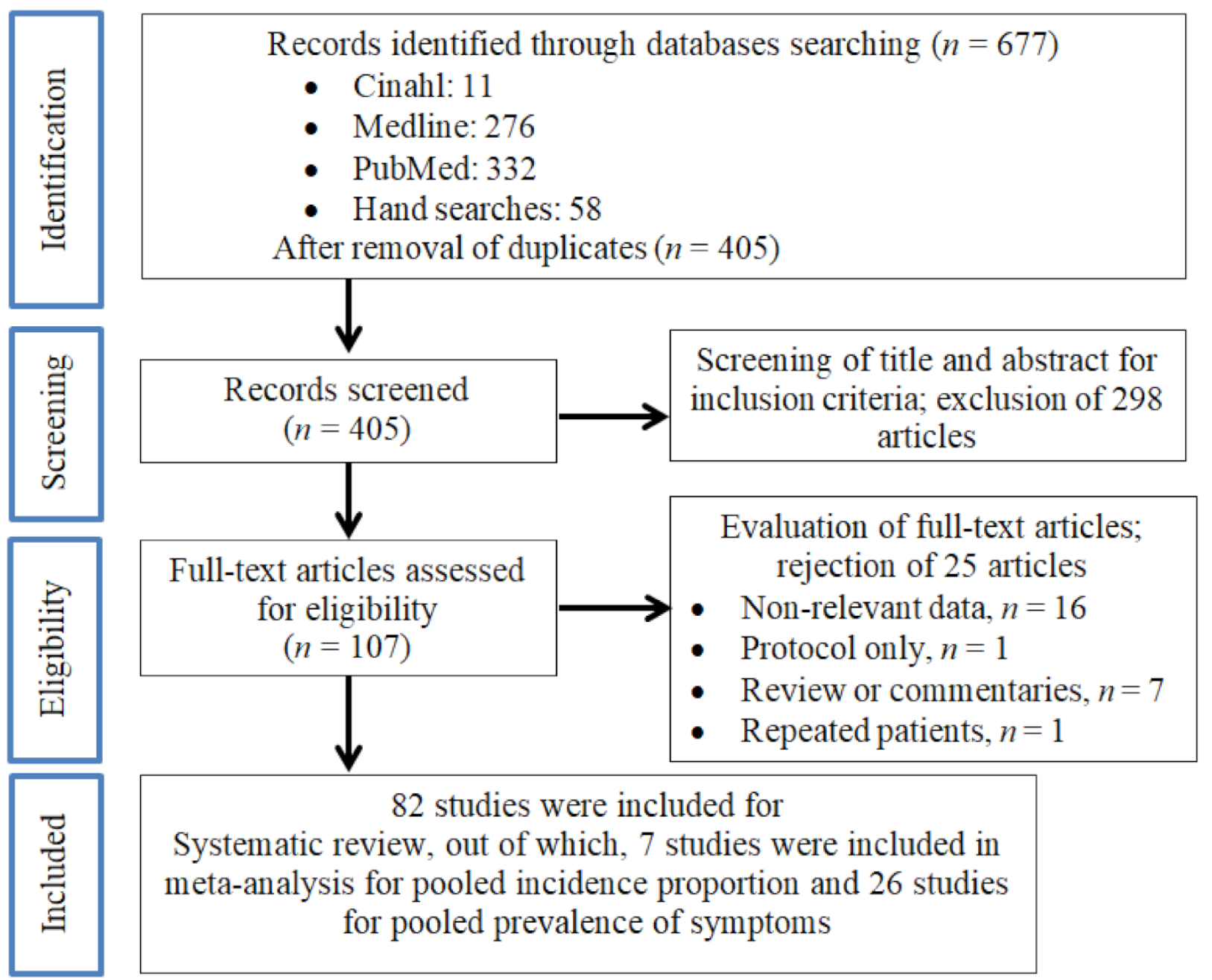

Figure 1. Preferred Reporting Items for Systematic Reviews and Meta-Analyses (PRISMA) flow diagram of the literature screening process.

\subsection{Incidence Proportion of COVID-19 among HIV Patients and Their Demographic Characteristics, Severity and Mortality}

The PIP of COVID-19 among PLHIV was 0.9\% (95\% CI 0.6\%, 1.1\%, $\mathrm{I}^{2}=98.5 \%$ ) based on the data gathered from seven cohort studies (Figure 2). The mean age of PLHIV coinfected with COVID-19 was 49.3 years \pm 10.7 . More than half $(n=3645)$ were female $(58.5 \%), 2483(39.8 \%)$ were male, 94 were of unknown gender $(1.5 \%)$, and $13(0.2 \%)$ were transgender. The mean body mass index (BMI) was $25.8 \pm 3.8(n=261$; range $=18.1-31.5)$. The severity of COVID-19 was known in 347 cases, of which $7.8 \%(27 / 347)$ were critical, $36.3 \%$ were severe (126/347), 32.8\% (114/347) were moderate, and $23.1 \%(80 / 347)$ were mild. Out of a total of 6235 cases, 1773 (28.4\%) needed hospitalization, of whom $2.3 \%$ $(218 / 1773)$ were severely ill and were admitted to the intensive care unit. The overall mortality rate among PLHIV co-infected with COVID-19 was 5.3\% (328/6235). 
Table 1. Characteristics of the included studies.

\begin{tabular}{|c|c|c|c|c|c|c|c|c|c|c|c|c|c|c|c|}
\hline \multirow{2}{*}{ No } & \multirow{2}{*}{ Author } & \multirow{2}{*}{ Year } & \multirow{2}{*}{ Country } & \multirow{2}{*}{$\begin{array}{l}\text { Study } \\
\text { Design }\end{array}$} & \multirow{2}{*}{$\begin{array}{c}\text { Quality } \\
\text { Assessment }\end{array}$} & \multirow{2}{*}{$\begin{array}{c}\text { HIV + } \\
\text { COVID-19 }\end{array}$} & \multirow{2}{*}{ Total HIV } & \multicolumn{4}{|c|}{ HIV + COVID-19 Patients Characteristics } & \multirow{2}{*}{$\begin{array}{c}\text { Summary of } \\
\text { Severity of } \\
\text { Infection at } \\
\text { Admission } \\
(\%)\end{array}$} & \multirow{2}{*}{$\begin{array}{c}\text { Number of HIV + } \\
\text { COVID-19 } \\
\text { Hospitalized (\%) }\end{array}$} & \multirow{2}{*}{$\begin{array}{l}\text { Number of Hiv + } \\
\text { COVID-19 } \\
\text { Admitted to } \\
\text { Intensive Care } \\
\text { Unit (\%) }\end{array}$} & \multirow{2}{*}{$\begin{array}{c}\text { Number of } \\
\text { Deaths } \\
\text { among HIV } \\
\text { COVID-19 } \\
(\%)\end{array}$} \\
\hline & & & & & & & & Average Age & $\begin{array}{c}\text { Average } \\
\text { BMI }\end{array}$ & Gender, $n(\%)$ & Ethnicity & & & & \\
\hline 1 & Adachi et al. [26] & 2020 & Japan & CS & $\begin{array}{c}\text { Supplementary } \\
\text { Table S3 }\end{array}$ & 2 & 2 & 38.7 & & $\begin{array}{l}\text { Transgender women, } \\
2(100)\end{array}$ & & & $2(100)$ & & \\
\hline 2 & Altuntas et al. [27] & 2020 & Turkey & CS & $\begin{array}{l}\text { Supplementary } \\
\text { Table S3 }\end{array}$ & 4 & 1224 & 43.5 & & Male, 4 (100) & & & $4(100)$ & $1(25)$ & $1(25)$ \\
\hline 3 & Baluku et al. [28] & 2020 & Uganda & $\mathrm{CR}$ & $\begin{array}{c}\text { Supplementary } \\
\text { Table S3 }\end{array}$ & 1 & 1 & 34.0 & & Female, 1 (100) & & & $1(100)$ & & \\
\hline 4 & Benkovic et al. [29] & 2020 & USA & CS & $\begin{array}{l}\text { Supplementary } \\
\text { Table S3 }\end{array}$ & 4 & 4 & 50.5 & & Male, 4 (100) & & & $1(25)$ & & \\
\hline 5 & Blanco et al. [30] & 2020 & Spain & CS & $\begin{array}{l}\text { Supplementary } \\
\text { Table S3 }\end{array}$ & 5 & 5 & 45.9 & & $\begin{array}{c}\text { Male, } 3(60) ; \\
\text { Transgender, } 2(40)\end{array}$ & & $\begin{array}{l}\text { Severe, } 2(40) ; \\
\text { Moderate, } 1 \\
\text { (20); Mild, } 2 \\
\text { (40) }\end{array}$ & $5(100)$ & $2(40)$ & \\
\hline 6 & Boulle et al. [31] & 2020 & $\begin{array}{l}\text { South } \\
\text { Africa }\end{array}$ & $\mathrm{RC}$ & Good & 3978 & 536,574 & & & $\begin{array}{l}\text { Male, } 877 \text { (22); } \\
\text { Female, } 3101 \text { (78) }\end{array}$ & & & $601(15.1)$ & & $115(2.9)$ \\
\hline 7 & Byrd et al. [32] & 2020 & USA & CS & $\begin{array}{l}\text { Supplementary } \\
\text { Table S3 }\end{array}$ & 27 & 27 & 53.0 & & $\begin{array}{c}\text { Male, } 21 \text { (77.8); } \\
\text { Female, 5 (18.5); } \\
\text { Transgender, 1 (3.7) }\end{array}$ & $\begin{array}{c}\text { Hispanic, 13; African } \\
\text { American, 6; White, } 7 \\
\text { Other, 1 }\end{array}$ & & $9(33.3)$ & & $1(3.7)$ \\
\hline 8 & Calza et al., b [33] & 2020 & Italy & CS & $\begin{array}{l}\text { Supplementary } \\
\text { Table S3 }\end{array}$ & 14 & 14 & 52.6 & & $\begin{array}{c}\text { Male, } 9(64.3) ; \\
\text { Female, } 5(35.7)\end{array}$ & White, 13; Other, 1 & & $3(21.4)$ & & \\
\hline 9 & Calza et al., a [34] & 2020 & Italy & CS & $\begin{array}{l}\text { Supplementary } \\
\text { Table S3 }\end{array}$ & 26 & 26 & 53.8 & & $\begin{array}{l}\text { Male, } 19(73.1) ; \\
\text { Female, } 7(26.9)\end{array}$ & Caucasian, 25; Unknown, 1 & & $5(19.2)$ & & \\
\hline 11 & Chiappe et al. [36] & 2020 & Peru & $C R$ & $\begin{array}{c}\text { Supplementary } \\
\text { Table S3 }\end{array}$ & 1 & 1 & 38.0 & & Male, $1(100)$ & & & & & $1(100)$ \\
\hline 12 & Childs et al. [37] & 2020 & UK & CS & $\begin{array}{c}\text { Supplementary } \\
\text { Table S3 }\end{array}$ & 18 & 18 & 52.0 & & $\begin{array}{l}\text { Male, } 12 \text { (66.7; } \\
\text { Female, } 6 \text { (33.3) }\end{array}$ & Black, 17; Non-black, 1 & & $18(100)$ & & $5(27.8)$ \\
\hline 13 & $\begin{array}{c}\text { Cipolat and Sprinz } \\
{[38]}\end{array}$ & 2020 & Brazil & $\mathrm{CR}$ & $\begin{array}{c}\text { Supplementary } \\
\text { Table S3 }\end{array}$ & 1 & 1 & 63.0 & & Female, $1(100)$ & & $\begin{array}{l}\text { Severe, } 1 \\
(100)\end{array}$ & $1(100)$ & & \\
\hline 14 & Coleman et al. [39] & 2020 & UK & $C R$ & $\begin{array}{l}\text { Supplementary } \\
\text { Table S3 }\end{array}$ & 1 & 1 & 55.0 & & Male, 1 (100) & & & $1(100)$ & $1(100)$ & \\
\hline 15 & Collins et al. [40] & 2020 & USA & CS & $\begin{array}{l}\text { Supplementary } \\
\text { Table S3 }\end{array}$ & 20 & 20 & 57.0 & 28.0 & $\begin{array}{l}\text { Male, } 13 \text { (65); Female, } \\
6(30) ; \text { Transgender, } \\
\text { (5) }\end{array}$ & $\begin{array}{l}\text { American African, 17; } \\
\text { White 1; Mixed race 1; } \\
\text { Hispanic/Latino1 }\end{array}$ & & $20(100)$ & & \\
\hline 16 & $\begin{array}{c}\text { Dandachi et al. } \\
{[41]}\end{array}$ & 2020 & USA & CS & $\begin{array}{l}\text { Supplementary } \\
\text { Table S3 }\end{array}$ & 286 & 286 & 51.4 & & $\begin{array}{l}\text { Male, } 212 \text { (74.1); } \\
\text { female; } 74(25.9)\end{array}$ & $\begin{array}{l}\text { African American, } 133, \\
\text { Hispanic, 78; White, } 48 ; \\
\text { Asian, 21; Unknown, } 6\end{array}$ & & $164(57.3)$ & $47(16.4)$ & $27(9.4)$ \\
\hline 17 & Del Amo et al. [42] & 2020 & Spain & Cohort & Good & 236 & 77,590 & & & $\begin{array}{l}\text { Male, } 204 \text { (86.4); } \\
\text { Female, } 32 \text { (13.6) }\end{array}$ & & & $151(64)$ & $15(6.4)$ & $20(8.5)$ \\
\hline 18 & $\begin{array}{l}\text { Di Biagio et al., a } \\
\text { [43] }\end{array}$ & 2020 & Italy & CS & $\begin{array}{l}\text { Supplementary } \\
\text { Table S3 }\end{array}$ & 4 & 1500 & 66.6 & & $\begin{array}{c}\text { Male, } 3 \text { (75); Female, } \\
1(25)\end{array}$ & & & $4(100)$ & & \\
\hline
\end{tabular}


Table 1. Cont.

\begin{tabular}{|c|c|c|c|c|c|c|c|c|c|c|c|c|c|c|c|}
\hline \multirow{2}{*}{ No } & \multirow{2}{*}{ Author } & \multirow{2}{*}{ Year } & \multirow{2}{*}{ Country } & \multirow{2}{*}{$\begin{array}{l}\text { Study } \\
\text { Design }\end{array}$} & \multirow{2}{*}{$\begin{array}{c}\text { Quality } \\
\text { Assessment }\end{array}$} & \multirow{2}{*}{$\begin{array}{c}\text { HIV + } \\
\text { COVID-19 }\end{array}$} & \multirow{2}{*}{ Total HIV } & \multicolumn{4}{|c|}{ HIV + COVID-19 Patients Characteristics } & \multirow{2}{*}{$\begin{array}{c}\text { Summary of } \\
\text { Severity of } \\
\text { Infection at } \\
\text { Admission } \\
(\%)\end{array}$} & \multirow{2}{*}{$\begin{array}{c}\text { Number of HIV + } \\
\text { COVVID-19 } \\
\text { Hospitalized (\%) }\end{array}$} & \multirow{2}{*}{$\begin{array}{l}\text { Number of Hiv + } \\
\text { COVID-19 } \\
\text { Admitted to } \\
\text { Intensive Care } \\
\text { Unit (\%) }\end{array}$} & \multirow{2}{*}{$\begin{array}{c}\text { Number of } \\
\text { Deaths } \\
\text { among HIV } \\
\text { COVIDI-19 } \\
(\%)\end{array}$} \\
\hline & & & & & & & & Average Age & $\begin{array}{c}\text { Average } \\
\text { BMI }\end{array}$ & Gender, $n(\%)$ & Ethnicity & & & & \\
\hline 19 & $\begin{array}{l}\text { Di Biagio et al., b } \\
{[44]}\end{array}$ & 2020 & Italy & CS & $\begin{array}{l}\text { Supplementary } \\
\text { Table S3 }\end{array}$ & 69 & 69 & 53.5 & & $\begin{array}{l}\text { Male, } 50 \text { (72.5); } \\
\text { Female; } 19 \text { (27.5) }\end{array}$ & Caucasian, 59; Others, 10 & $\begin{array}{l}\text { Moderate- } \\
\text { Severe, } 38 \\
\text { (55.1) }\end{array}$ & $38(55.1)$ & & $7(10.1)$ \\
\hline 20 & $\begin{array}{l}\text { Di Giambenedetto } \\
\text { et al. [45] }\end{array}$ & 2020 & Italy & $\mathrm{CR}$ & $\begin{array}{l}\text { Supplementary } \\
\text { Table S3 }\end{array}$ & 1 & 1 & 75.0 & & Male, 1 (100) & & $\begin{array}{c}\text { Severe, } 1 \\
(100)\end{array}$ & $1(100)$ & $1(100)$ & \\
\hline 21 & D'Ettorre et al. [46] & 2020 & Italy & $\mathrm{CR}$ & $\begin{array}{c}\text { Supplementary } \\
\text { Table S3 }\end{array}$ & 1 & 1 & 52.0 & & Female, 1 (100) & Ethiopian, 1 & $\begin{array}{c}\text { Severe, } 1 \\
(100)\end{array}$ & $1(100)$ & & \\
\hline 22 & Elhadi et al. [47] & 2020 & Libya & $\mathrm{CR}$ & $\begin{array}{c}\text { Supplementary } \\
\text { Table S3 }\end{array}$ & 1 & 1 & 86.0 & & Female, 1 (100) & & & $1(100)$ & $1(100)$ & $1(100)$ \\
\hline 23 & Etienne et al. [48] & 2020 & France & $\mathrm{PC}$ & Poor & 54 & 54 & 54.0 & 25.2 & $\begin{array}{l}\text { Male, } 33(61.1) \\
\text { Female, } 21 \text { (38.9) }\end{array}$ & $\begin{array}{c}\text { African, 26; South } \\
\text { American, 3; European, } 25\end{array}$ & $\begin{array}{l}\text { Moderate, } 35 \\
\text { (64.8); severe, } \\
14 \text { (25.9); } \\
\text { critical, } \\
(9.3)\end{array}$ & & & $1(1.9)$ \\
\hline 24 & $\begin{array}{c}\text { Faranacci et al. } \\
{[49]}\end{array}$ & 2020 & Italy & $\mathrm{CR}$ & $\begin{array}{c}\text { Supplementary } \\
\text { Table S3 }\end{array}$ & 1 & 1 & 59.0 & & Male, $1(100)$ & & & $1(100)$ & $1(100)$ & $1(100)$ \\
\hline 25 & Gadelha et al. [50] & 2020 & Brazil & CS & $\begin{array}{c}\text { Supplementary } \\
\text { Table S3 }\end{array}$ & 2 & 2 & 53.0 & & Male, 2 (100) & & & $2(100)$ & $1(50)$ & \\
\hline 26 & Geretti et al. [51] & 2020 & UK & PC & Good & 115 & 115 & 55.0 & & $\begin{array}{l}\text { Male, } 76(66.1) ; \\
\text { Female } 39 \text { (33.9) }\end{array}$ & $\begin{array}{l}\text { White, 44; Black, 48; Asian, } \\
1 \text {; Other, 13; Unknown, } 9\end{array}$ & & & & $26(22.6)$ \\
\hline 27 & $\begin{array}{c}\text { Gervasoni et al. } \\
\text { [52] }\end{array}$ & 2020 & Italy & CS & $\begin{array}{c}\text { Supplementary } \\
\text { Table S3 }\end{array}$ & 47 & 47 & 51.0 & & Unknown, 47 (100) & & & $13(27.7)$ & & $2(4.3)$ \\
\hline 29 & Guo et al. [54] & 2020 & China & CS & $\begin{array}{l}\text { Supplementary } \\
\text { Table S3 }\end{array}$ & 14 & 14 & 45.3 & & $\begin{array}{l}\text { Male, } 13(92.9) ; \\
\text { Female 1 (7.1) }\end{array}$ & & 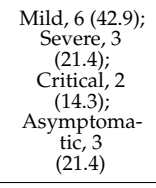 & $14(100)$ & $1(7.1)$ & $2(14.3)$ \\
\hline 30 & Haddad et al. [55] & 2020 & USA & $\mathrm{CR}$ & $\begin{array}{c}\text { Supplementary } \\
\text { Table S3 }\end{array}$ & 1 & 1 & 41.0 & & Male, $1(100)$ & & & $1(100)$ & $1(100)$ & \\
\hline 31 & Hadi et al. [56] & 2020 & USA & CS & $\begin{array}{l}\text { Supplementary } \\
\text { Table S3 }\end{array}$ & 404 & 404 & 48.2 & & $\begin{array}{l}\text { Male, } 285(70.5) ; \\
\text { Female, } 119 \text { (29.5) }\end{array}$ & $\begin{array}{c}\text { African America, 201; } \\
\text { White, 137; } \\
\text { Hispanic/Latino53; Asian, } \\
\text { 10; Others, 3 } \\
\end{array}$ & & $78(19.3)$ & $27(6.7)$ & \\
\hline 32 & Harter et al. [57] & 2020 & Germany & CS & $\begin{array}{l}\text { Supplementary } \\
\text { Table S3 }\end{array}$ & 33 & 33 & 47.5 & & $\begin{array}{l}\text { Male, } 30 \text { (90.9); } \\
\text { Female, 3 }(9.1)\end{array}$ & & $\begin{array}{c}\text { Mild, } 25 \\
\text { (75.8); Severe, } \\
2 \text { (6.1); } \\
\text { Critical, } 6 \\
\text { (18.2) }\end{array}$ & $14(42.4)$ & $6(18.2)$ & $3(9.1)$ \\
\hline 33 & Ho et al. [58] & 2020 & USA & CS & $\begin{array}{l}\text { Supplementary } \\
\text { Table S3 }\end{array}$ & 93 & 93 & 58.0 & 26.7 & $\begin{array}{c}\text { Male, } 67(72.1) \\
\text { Female, } 23(24.7) ; \\
\text { Transgender, } 3(3.2)\end{array}$ & $\begin{array}{l}\text { White, 21; Black 38; } \\
\text { Unknown, } 34\end{array}$ & & $72(77.4)$ & $19(20.4)$ & $19(20.4)$ \\
\hline
\end{tabular}


Table 1. Cont.

\begin{tabular}{|c|c|c|c|c|c|c|c|c|c|c|c|c|c|c|c|}
\hline \multirow{2}{*}{ No } & \multirow{2}{*}{ Author } & \multirow{2}{*}{ Year } & \multirow{2}{*}{ Country } & \multirow{2}{*}{$\begin{array}{l}\text { Study } \\
\text { Design }\end{array}$} & \multirow{2}{*}{$\begin{array}{c}\text { Quality } \\
\text { Assessment }\end{array}$} & \multirow{2}{*}{$\begin{array}{c}\text { HIV + } \\
\text { COVID-19 }\end{array}$} & \multirow{2}{*}{ Total HIV } & \multicolumn{4}{|c|}{ HIV + COVID-19 Patients Characteristics } & \multirow{2}{*}{$\begin{array}{c}\text { Summary of } \\
\text { Severity of } \\
\text { Infection at } \\
\text { Admission } \\
(\%)\end{array}$} & \multirow{2}{*}{$\begin{array}{c}\text { Number of HIV + } \\
\text { COVID-19 } \\
\text { Hospitalized (\%) }\end{array}$} & \multirow{2}{*}{$\begin{array}{c}\text { Number of Hiv + } \\
\text { COVID-19 } \\
\text { Admitted to } \\
\text { Intensive Care } \\
\text { Unit (\%) }\end{array}$} & \multirow{2}{*}{$\begin{array}{c}\text { Number of } \\
\text { Deaths } \\
\text { among HIV } \\
\text { COVIID-19 } \\
(\%)\end{array}$} \\
\hline & & & & & & & & Average Age & $\begin{array}{c}\text { Average } \\
\text { BMI }\end{array}$ & Gender, $n(\%)$ & Ethnicity & & & & \\
\hline 34 & Hu et al. [59] & 2020 & China & CS & $\begin{array}{l}\text { Supplementary } \\
\text { Table S3 }\end{array}$ & 12 & 5953 & 42.4 & & $\begin{array}{l}\text { Male, } 10(83.3) ; \\
\text { Female, } 2(16.7)\end{array}$ & & $\begin{array}{c}\text { Mild, } 9 \text { (75.0); } \\
\text { Severe, 2 } \\
\text { (16.7); Fatal,1 } \\
\text { (8.3) }\end{array}$ & $9(75)$ & $2(16.7)$ & $1(8.3)$ \\
\hline 35 & Huang et al. [60] & 2020 & China & Cohort & Good & 35 & 6001 & 52.0 & & $\begin{array}{l}\text { Male, } 33 \text { (94.3); } \\
\text { Female, } 2 \text { (5.7) }\end{array}$ & & $\begin{array}{l}\text { Severe, } 15 \\
(42.9)\end{array}$ & & & $2(5.7)$ \\
\hline 36 & Inciarte et al. [23] & 2020 & Spain & PC & Poor & 53 & 5683 & 44.0 & & $\begin{array}{l}\text { Male, } 45(84.9) ; \\
\text { Female, } 8(15.1)\end{array}$ & & $\begin{array}{c}\text { Severe, } 6 \\
(11.3)\end{array}$ & $26(49.1)$ & $4(7.5)$ & $2(3.8)$ \\
\hline 37 & Iordanou et al. [61] & 2020 & Cyprus & CR & $\begin{array}{l}\text { Supplementary } \\
\text { Table S3 }\end{array}$ & 1 & 1 & 58.0 & & Male, 1 (100) & Caucasian, 1 & $\begin{array}{c}\text { Severe, } 1 \\
(100)\end{array}$ & $1(100)$ & $1(100)$ & \\
\hline 38 & Isernia et al. [62] & 2020 & France & CS & $\begin{array}{l}\text { Supplementary } \\
\text { Table S3 }\end{array}$ & 24 & 30 & & & Unknown, 24 (100) & & & & & $2(8.3)$ \\
\hline 39 & Karmen et al. [63] & 2020 & USA & $\mathrm{RC}$ & Poor & 21 & 21 & & & Unknown, 21 (100) & & & $21(100)$ & $6(28.6)$ & $6(28.6)$ \\
\hline 40 & Khaba et al. [64] & 2020 & $\begin{array}{l}\text { South } \\
\text { Africa }\end{array}$ & CR & $\begin{array}{l}\text { Supplementary } \\
\text { Table S3 }\end{array}$ & 1 & $1+$ & 19.0 & 18.1 & Male, $1(100)$ & African, 1 & & $1(100)$ & $1(100)$ & $1(100)$ \\
\hline 41 & Kim et al. [65] & 2020 & $\begin{array}{l}\text { South } \\
\text { Korea }\end{array}$ & $\mathrm{CR}$ & $\begin{array}{l}\text { Supplementary } \\
\text { Table S3 }\end{array}$ & 1 & 1 & 29.0 & 24.2 & Male, 1 (100) & Korean, 1 & & & & \\
\hline 42 & Kumar et al. [66] & 2020 & USA & CR & $\begin{array}{c}\text { Supplementary } \\
\text { Table S3 }\end{array}$ & 1 & 1 & 50.0 & & Male, 1 (100) & African American, 1 & & & & \\
\hline 43 & Li et al. [67] & 2020 & China & CS & $\begin{array}{l}\text { Supplementary } \\
\text { Table S3 }\end{array}$ & 2 & 2 & 54.8 & & Male, 2 (100) & & & $2(100)$ & & \\
\hline 45 & Madge et al. [69] & 2020 & UK & CS & $\begin{array}{l}\text { Supplementary } \\
\text { Table S3 }\end{array}$ & 18 & 18 & 63.0 & & $\begin{array}{l}\text { Male, } 14(77.8) ; \\
\text { Female, } 4 \text { (22.2) }\end{array}$ & $\begin{array}{l}\text { Black, Asian and Minority, } \\
\text { 9; Unknown, } 9\end{array}$ & & $18(100)$ & $2(11.1)$ & $3(16.7)$ \\
\hline 46 & $\begin{array}{c}\text { Maggiolo et al. } \\
{[24]}\end{array}$ & 2020 & Italy & PC & Good & 55 & 2898 & 54.0 & & $\begin{array}{c}\text { Male, } 44(80) ; \text { Female, } \\
11(20)\end{array}$ & & & $15(27.3)$ & & $4(7.3)$ \\
\hline 47 & Mang et al. [70] & 2020 & Germany & CR & $\begin{array}{c}\text { Supplementary } \\
\text { Table S3 }\end{array}$ & 1 & 1 & 52.0 & & Male, 1 (100) & & & $1(100)$ & $1(100)$ & \\
\hline 48 & $\underset{[71]}{\text { Marimuthu et al. }}$ & 2020 & India & CS & $\begin{array}{l}\text { Supplementary } \\
\text { Table S3 }\end{array}$ & 6 & 6 & 39.9 & & $\begin{array}{l}\text { Male, } 3 \text { (50); Female, } \\
2 \text { (33.3), Transgender, } \\
1 \text { (16.7) }\end{array}$ & & & & & \\
\hline 49 & $\begin{array}{c}\text { Meyerowitz et al. } \\
{[72]}\end{array}$ & 2020 & USA & CS & $\begin{array}{l}\text { Supplementary } \\
\text { Table S3 }\end{array}$ & 36 & 36 & 48.1 & & $\begin{array}{l}\text { Male, } 21 \text { (58.3); } \\
\text { Female, } 15(41.7)\end{array}$ & $\begin{array}{l}\text { African American, 16; } \\
\text { Hispanic, 12; Others, } 8\end{array}$ & $\begin{array}{l}\text { Severe, } 8 \\
(22.2) ; \\
\text { Critical, } \\
(19.4)\end{array}$ & $21(58.3)$ & $5(13.9)$ & $2(5.6)$ \\
\hline 50 & $\begin{array}{l}\text { Miyashita and } \\
\text { Kuno [73] }\end{array}$ & 2020 & USA & CS & $\begin{array}{l}\text { Supplementary } \\
\text { Table S3 }\end{array}$ & 161 & 161 & & & $\begin{array}{l}\text { Male, } 125(77.6) ; \\
\text { Female, } 36(22.4)\end{array}$ & & & $161(100)$ & $36(22.4)$ & $23(14.3)$ \\
\hline 51 & Modi et al. [74] & 2020 & USA & CR & $\begin{array}{l}\text { Supplementary } \\
\text { Table S3 }\end{array}$ & 1 & 1 & 32.0 & & Unknown, 1 (100) & African American, 1 & Mild, 1 (100) & $1(100)$ & & \\
\hline 52 & Molina et al. [75] & 2020 & Spain & $\mathrm{RC}$ & Poor & 8 & 902 & 45.1 & & $\begin{array}{l}\text { Male, } 7(87.5) ; \\
\text { Female, 1(12.5) }\end{array}$ & & $\begin{array}{l}\text { Mild, } 5 \text { (62.5); } \\
\text { Severe, } \\
(37.5)\end{array}$ & & & $1(12.5)$ \\
\hline
\end{tabular}


Table 1. Cont.

\begin{tabular}{|c|c|c|c|c|c|c|c|c|c|c|c|c|c|c|c|}
\hline \multirow{2}{*}{ No } & \multirow{2}{*}{ Author } & \multirow{2}{*}{ Year } & \multirow{2}{*}{ Country } & \multirow{2}{*}{$\begin{array}{l}\text { Study } \\
\text { Design }\end{array}$} & \multirow{2}{*}{$\begin{array}{c}\text { Quality } \\
\text { Assessment }\end{array}$} & \multirow{2}{*}{$\begin{array}{c}\text { HIV + } \\
\text { COVID-19 }\end{array}$} & \multirow{2}{*}{ Total HIV } & \multicolumn{4}{|c|}{ HIV + COVID-19 Patients Characteristics } & \multirow{2}{*}{$\begin{array}{l}\text { Summary of } \\
\text { Severity of } \\
\text { Infection at } \\
\text { Admission } \\
\text { (\%) }\end{array}$} & \multirow{2}{*}{$\begin{array}{c}\text { Number of HIV + } \\
\text { COVID-19 } \\
\text { Hospitalized (\%) }\end{array}$} & \multirow{2}{*}{$\begin{array}{l}\text { Number of Hiv + } \\
\text { COVID-19 } \\
\text { Admitted to } \\
\text { Intensive Care } \\
\text { Unit (\%) }\end{array}$} & \multirow{2}{*}{$\begin{array}{c}\text { Number of } \\
\text { Deaths } \\
\text { among HIV } \\
\text { COVID-19 } \\
(\%)\end{array}$} \\
\hline & & & & & & & & Average Age & $\begin{array}{c}\text { Average } \\
\text { BMI }\end{array}$ & Gender, $n(\%)$ & Ethnicity & & & & \\
\hline 53 & Mondi et al. [76] & 2020 & Italy & CS & $\begin{array}{l}\text { Supplementary } \\
\text { Table S3 }\end{array}$ & 5 & 5 & 46.2 & & $\begin{array}{c}\text { Male, } 4 \text { (80); } \\
\text { Transgender, } 1 \text { (20) }\end{array}$ & & $\begin{array}{l}\text { Mild, } 3(60) ; \\
\text { Severe, 1 (20); } \\
\text { Asymptoma- } \\
\text { tic 1 } \\
\text { (20) }\end{array}$ & & & \\
\hline 54 & $\begin{array}{c}\text { Nakamoto et al. } \\
{[77]}\end{array}$ & 2020 & Japan & $\mathrm{CR}$ & $\begin{array}{l}\text { Supplementary } \\
\text { Table S3 }\end{array}$ & 1 & 1 & 28.0 & & Male, 1 (100) & & & $1(100)$ & & \\
\hline 55 & Okoh et al. [78] & 2020 & USA & CS & $\begin{array}{l}\text { Supplementary } \\
\text { Table S3 }\end{array}$ & 27 & 27 & 58.0 & 31.5 & $\begin{array}{l}\text { Male, } 15 \text { (55.6); } \\
\text { Female, 12 (44.4) }\end{array}$ & $\begin{array}{l}\text { African American, 25; } \\
\text { Hispanic, 2 }\end{array}$ & & $13(48.1)$ & $3(11.1)$ & $2(7.4)$ \\
\hline 57 & Parker et al., b [80] & 2020 & $\begin{array}{l}\text { South } \\
\text { Africa }\end{array}$ & CS & $\begin{array}{l}\text { Supplementary } \\
\text { Table S3 }\end{array}$ & 24 & 24 & 46.2 & & $\begin{array}{c}\text { Male, 6 (25); Female, } \\
18 \text { (75) }\end{array}$ & & & $19(79.2)$ & $5(20.8)$ & $6(25)$ \\
\hline 58 & Patel and Pella [81] & 2020 & USA & $\mathrm{CR}$ & $\begin{array}{l}\text { Supplementary } \\
\text { Table S3 }\end{array}$ & 1 & 1 & 58.0 & & Male, 1 (100) & & & $1(100)$ & & \\
\hline 59 & $\begin{array}{c}\text { Przydzial et al. } \\
{[82]}\end{array}$ & 2020 & USA & CS & $\begin{array}{l}\text { Supplementary } \\
\text { Table S3 }\end{array}$ & 2 & 2 & 46.5 & & Male, 2 (100) & & & $2(100)$ & & \\
\hline 60 & Qasim et al. [83] & 2020 & USA & CR & $\begin{array}{l}\text { Supplementary } \\
\text { Table S3 } \\
\end{array}$ & 1 & 1 & 37.0 & & Male, 1 (100) & & Mild, 1 (100) & $1(100)$ & & \\
\hline 61 & Ridgway et al. [84] & 2020 & USA & CS & $\begin{array}{l}\text { Supplementary } \\
\text { Table S3 }\end{array}$ & 5 & 5 & 56.1 & & $\begin{array}{l}\text { Male, } 1 \text { (20); Female, } \\
4(80)\end{array}$ & & Mild, 5 (100) & $5(100)$ & & \\
\hline 63 & Rivas et al. [86] & 2020 & Panama & CS & $\begin{array}{l}\text { Supplementary } \\
\text { Table S3 }\end{array}$ & 2 & 2 & 54.9 & & Male, 2 (100) & & & $2(100)$ & $1(50)$ & \\
\hline 64 & Ruan et al. [87] & 2020 & China & CS & $\begin{array}{l}\text { Supplementary } \\
\text { Table S3 }\end{array}$ & 4 & 4 & 55.8 & & Male, 4 (100) & & $\begin{array}{l}\text { Moderate, } 2 \\
\text { (50); Severe, } 2 \\
\text { (50) }\end{array}$ & $4(100)$ & & \\
\hline 65 & Sasset et al. [88] & 2020 & Italy & CS & $\begin{array}{l}\text { Supplementary } \\
\text { Table S3 }\end{array}$ & 2 & 2 & 54.1 & & Male, 2 (100) & & $\begin{array}{l}\text { Severe, } 2 \\
(100)\end{array}$ & $2(100)$ & $2(100)$ & \\
\hline 66 & Shalev et al. [89] & 2020 & USA & CS & $\begin{array}{l}\text { Supplementary } \\
\text { Table S3 }\end{array}$ & 31 & 31 & 60.7 & & Male, 24; Female, 7 & $\begin{array}{l}\text { Black, } 16 ; \text { White, } 5 ; \\
\text { Hispanic, } 9 \text {; unknown, } 1\end{array}$ & $\begin{array}{l}\text { Mild, 1 (3.2); } \\
\text { Moderate, 2 } \\
\text { (6.5); Severe, } \\
\text { 21 (67.7); } \\
\text { Critical, } 7 \\
\text { (22.6) }\end{array}$ & & & $8(25.8)$ \\
\hline 67 & Shekhar et al. [90] & 2020 & USA & CS & $\begin{array}{l}\text { Supplementary } \\
\text { Table S3 }\end{array}$ & 5 & 5 & 47.4 & & $\begin{array}{c}\text { Male, } 4 \text { (80); Female, } \\
1 \text { (20) }\end{array}$ & & & $3(60)$ & $1(20)$ & \\
\hline 68 & Sigel et al. [91] & 2020 & USA & CS & $\begin{array}{l}\text { Supplementary } \\
\text { Table S3 }\end{array}$ & 88 & 88 & 61.0 & & $\begin{array}{l}\text { Male, } 66 \text { (75); Female, } \\
22(25)\end{array}$ & $\begin{array}{l}\text { White, 17; Black, 35; } \\
\text { Hispanic, 26; Other, } 10\end{array}$ & $\begin{array}{c}\text { Mild, } 16 \\
\text { (18.2); } \\
\text { Moderate, } 54 \\
\text { (61.4); Severe, } \\
\text { 18 (20.5) }\end{array}$ & $88(100)$ & & $18(20.5)$ \\
\hline 69 & Stoeckle et al. [92] & 2020 & USA & $\mathrm{RC}$ & Good & 30 & 30 & 60.5 & 27.2 & $\begin{array}{c}\text { Male, } 24 \text { (80); Female } \\
6(20)\end{array}$ & $\begin{array}{l}\text { White, 8; Black, 9; Other, 6; } \\
\text { Not specified, } 7\end{array}$ & & $30(100)$ & $4(13.3)$ & $2(6.7)$ \\
\hline 70 & Su et al. [93] & 2020 & China & CR & $\begin{array}{l}\text { Supplementary } \\
\text { Table S3 }\end{array}$ & 1 & 1 & 32.0 & & Male, 1 (100) & & & & & \\
\hline
\end{tabular}


Table 1. Cont.

\begin{tabular}{|c|c|c|c|c|c|c|c|c|c|c|c|c|c|c|c|}
\hline \multirow{2}{*}{ No } & \multirow{2}{*}{ Author } & \multirow{2}{*}{ Year } & \multirow{2}{*}{ Country } & \multirow{2}{*}{$\begin{array}{l}\text { Study } \\
\text { Design }\end{array}$} & \multirow{2}{*}{$\begin{array}{c}\text { Quality } \\
\text { Assessment }\end{array}$} & \multirow{2}{*}{$\begin{array}{c}\text { HIV + } \\
\text { COVID-19 }\end{array}$} & \multirow{2}{*}{ Total HIV } & \multicolumn{4}{|c|}{ HIV + COVID-19 Patients Characteristics } & \multirow{2}{*}{$\begin{array}{l}\text { Summary of } \\
\text { Severity of } \\
\text { Infection at } \\
\text { Admission } \\
\text { (\%) }\end{array}$} & \multirow{2}{*}{$\begin{array}{c}\text { Number of HIV + } \\
\text { COVID-19 } \\
\text { Hospitalized (\%) }\end{array}$} & \multirow{2}{*}{$\begin{array}{c}\text { Number of Hiv + } \\
\text { COVIID-19 } \\
\text { Admitted to } \\
\text { Intensive Care } \\
\text { Unit (\%) }\end{array}$} & \multirow{2}{*}{$\begin{array}{c}\text { Number of } \\
\text { Deaths } \\
\text { among HIV } \\
\text { COVIII-19 } \\
(\%)\end{array}$} \\
\hline & & & & & & & & Average Age & $\begin{array}{c}\text { Average } \\
\text { BMI }\end{array}$ & Gender, $n(\%)$ & Ethnicity & & & & \\
\hline 71 & Sun et al. [94] & 2020 & Singapore & $\mathrm{CR}$ & $\begin{array}{l}\text { Supplementary } \\
\text { Table S3 }\end{array}$ & 1 & 1 & 37.0 & & Male, $1(100)$ & & Mild, $1(100)$ & $1(100)$ & & \\
\hline 72 & $\begin{array}{c}\text { Suwanwongse and } \\
\text { Shabarek, a [95] }\end{array}$ & 2020 & USA & CS & $\begin{array}{c}\text { Supplementary } \\
\text { Table S3 }\end{array}$ & 9 & 9 & 46.6 & & $\begin{array}{l}\text { Male, } 7 \text { (77.8); } \\
\text { Female, } 2 \text { (22.2) }\end{array}$ & & & $9(100)$ & $6(66.7)$ & $7(77.8)$ \\
\hline 73 & $\begin{array}{l}\text { Suwanwongse and } \\
\text { Shabarek, b [96] }\end{array}$ & 2020 & USA & CS & $\begin{array}{c}\text { Supplementary } \\
\text { Table S3 }\end{array}$ & 5 & 5 & 47.1 & & $\begin{array}{c}\text { Male, } 4 \text { (80); Female, } \\
1(20)\end{array}$ & & & $5(1000)$ & $3(60)$ & $1(20)$ \\
\hline 74 & Toombs et al. [97] & 2020 & UK & CS & $\begin{array}{c}\text { Supplementary } \\
\text { Table S3 }\end{array}$ & 3 & 3 & 46.5 & & $\begin{array}{l}\text { Male, } 2 \text { (66.7); } \\
\text { Female, } 1 \text { (33.3) }\end{array}$ & & & $3(100)$ & $1(33.3)$ & $1(33.3)$ \\
\hline 75 & Vizcarra et al. [25] & 2020 & Spain & PC & Good & 35 & 2873 & 53.6 & 25.5 & $\begin{array}{l}\text { Male, } 30 \text { (85.7); } \\
\text { Female, } 5 \text { (14.3) }\end{array}$ & & & $35(100)$ & $6(17.1)$ & \\
\hline 76 & Wang et al. [98] & 2020 & China & CR & $\begin{array}{c}\text { Supplementary } \\
\text { Table S3 }\end{array}$ & 1 & 1 & 37.0 & & Unknown, 1 (100) & & & $1(100)$ & & \\
\hline 77 & Wu et al., a [99] & 2020 & China & CR & $\begin{array}{c}\text { Supplementary } \\
\text { Table S3 }\end{array}$ & 1 & 1 & 49.0 & & Female, 1 (100) & & $\begin{array}{l}\text { Moderate, } 1 \\
(100)\end{array}$ & $1(100)$ & & \\
\hline 78 & Wu et al., b [100] & 2020 & China & CS & $\begin{array}{c}\text { Supplementary } \\
\text { Table S3 }\end{array}$ & 2 & 2 & 49.5 & & Male, 2 (100) & & & $2(100)$ & & \\
\hline 79 & $\underset{[101]}{\text { Yamamoto al. }}$ & 2020 & Japan & CS & $\begin{array}{l}\text { Supplementary } \\
\text { Table S3 }\end{array}$ & 5 & 5 & 49.5 & & $\begin{array}{c}\text { Male, } 3(60) ; \\
\text { Transgender women, } \\
2(40)\end{array}$ & & Mild, 5 (100) & $5(100)$ & & \\
\hline 80 & Zhang et al. [102] & 2020 & China & CS & $\begin{array}{l}\text { Supplementary } \\
\text { Table S3 }\end{array}$ & 2 & 2 & 49.5 & & Male, 2 (100) & & & $2(100)$ & & \\
\hline 82 & Zhu et al. [104] & 2020 & China & $\mathrm{CR}$ & $\begin{array}{l}\text { Supplementary } \\
\text { Table S3 }\end{array}$ & 1 & 1 & 61.0 & & Male, 1 (100) & & & $1(100)$ & & \\
\hline
\end{tabular}

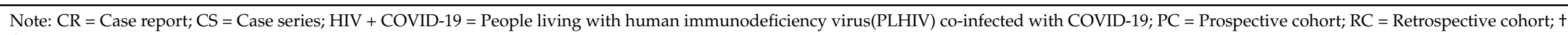
Autopsy case. 


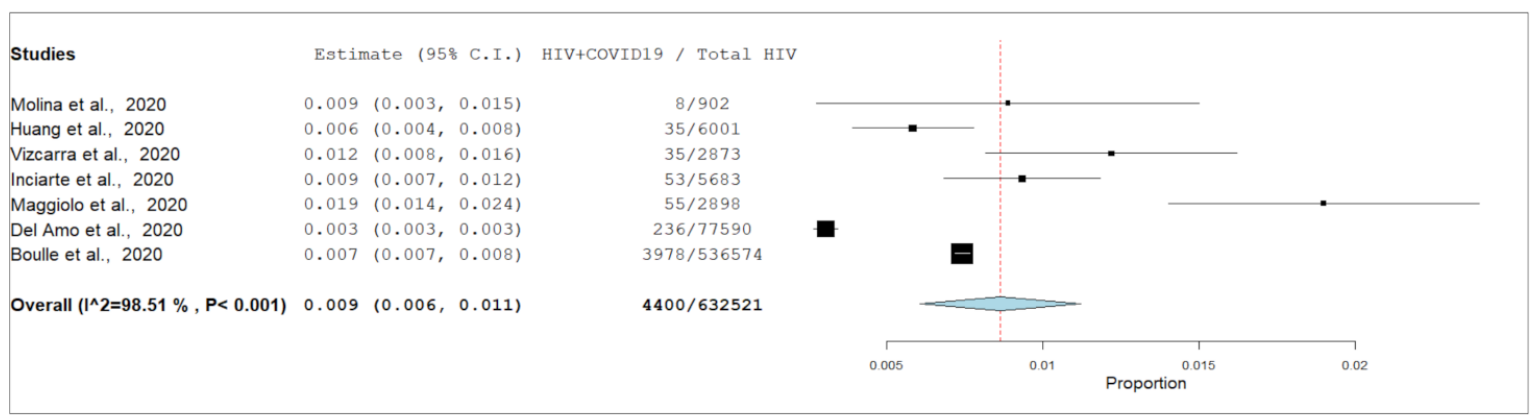

Figure 2. Pooled incidence proportion of COVID-19 among PLHIV based on data accumulated from cohort studies.

\subsection{Comorbidities among PLHIV Co-Infected with COVID-19}

As shown in Supplementary Table S5, cardiovascular disease (27.2\%) was the most frequent comorbidity, of which hypertension accounted for $23.9 \%$. This was followed by diabetes mellitus (12.2\%), chronic lung disease and asthma ( $4.2 \%$ each), and lifestyle-related disorders comprising dyslipidaemia and obesity (5.4\%), smoking, alcoholism, and illicit drug intake $(2.9 \%, 0.7 \%$, and $1.3 \%$ respectively). Other comorbidities of note are chronic kidney $(6.4 \%)$ and liver disorders $(1.4 \%)$.

\subsection{HIV Profile of PLHIV Co-Infected with COVID-19}

The diagnosis of HIV was made between 1988 and 2020; the mean duration of infection ranged from under 1 year to 30 years. Anti-retroviral treatment (ART) was documented in 1253 out of $6235(20.1 \%)$ of cases, with a mean duration of receiving antiretroviral treatment ranging from 2 to 23 years. Ten patients were newly diagnosed, thus treatment naïve, and another 9 patients were not on treatment for unspecified reasons. Nucleoside reverse transcriptase inhibitor (NRTI) was the most frequently prescribed ART for the HIV patients, followed by integrase strand transfer inhibitor, non-nucleoside reverse transcriptase inhibitors, and protease inhibitors.

The CD4 cell counts before diagnosis of COVID-19 were available in only 844 out of 6235 cases; the nadir CD4 cell count ranged from 97 to 434 in these cases. Out of the 844 cases, 115 had counts of $<350$ cells / uL while the remaining 729 patients had counts of $\geq 350$ cells $/$ uL.

Using the World Health Organization (WHO) HIV / AIDS revised clinical staging [20], 354 out of $6235(5.7 \%)$ patients met the criteria for advanced disease, 115 of which were defined by immunological criteria (CD4 cell count $<350 \mu \mathrm{L}$ ), and 239 by clinical criteria (clinical Stage 3 or 4). Results on the viral load measurements pre-COVID was available in $1023 \mathrm{HIV}$ patients of whom 294 had undetectable viral load (<20 RNA copies/mL) and 729 had $>20$ RNA copies/mL. Details on the HIV profiles are provided in Supplementary Table S6.

\subsection{Vital Signs, Results of COVID-19 Testing and Chest Imaging Findings}

The majority of studies ( $n=5662$ patients) used nucleic acid-based testing to diagnose COVID-19. A total of 5522 patients (97.5\%) tested positive. In 125 patients $(2.2 \%)$, the results of nucleic acid testing were not known, and 15 cases $(0.3 \%)$ tested negative. Interestingly, six cases negative for the nucleic acid-based test were seropositive for antibodies to COVID19. The method to diagnose COVID-19 was not known in 496 cases, of which 87 were reported as positive cases. Another observation is that 46 cases had at least one negative result (up to maximum 7 negative results) prior to conversion to positivity for the nucleic acid-based test despite the presence of multiple symptoms related to the infection.

COVID-19 antibody (IgM and/or IgG) testing was done in 89 patients. The results demonstrated that some cases positive for the virus nucleic acid either remained persistently 
seronegative $(n=11)$ or had a prolonged viral shedding duration. In the latter, 8 cases who were IgM positive continued viral RNA shedding from beyond day 14 up to 41 days, while 6 cases who were IgG positive continued to shed from 15 to 32 days.

On presentation, the body temperature ranged from 35.9 to $40{ }^{\circ} \mathrm{C}$ and the systolic and diastolic blood pressure from 93 to $172 \mathrm{mmHg}$ and 33 to $97 \mathrm{mmHg}$, respectively. Pulse rate and respiration rate ranged from 57 to 160 beats/minute and 14 to 40 breaths/minute, respectively. Oxygen saturation when breathing ambient air ranged from 56 to $100 \mathrm{mmHg}$; in 27 cases, the oxygen saturation was below $90 \%$ (hypoxaemia). $\mathrm{PaO}_{2} / \mathrm{FiO}_{2}$ ratio ranged from 50 to $500 \mathrm{mmHg}$; about $50 \%$ of the cases had mild to severe hypoxemia (refer to Supplementary Table S7 for details).

\subsection{Treatment for COVID-19}

Details of the treatments given to PLHIV co-infected with COVID-19 are given in Supplementary Table S8. Information regarding pharmacological intervention was recorded in 1429 patients. Hydroxychloroquine was given to 413 (28.9\%) patients. Eighty-three patients received anti-virals which included Oseltamivir, Arbidol, or interferon-alpha $(n=41,2.9 \%)$ and Remdesivir $(n=27,1.9 \%)$. Various antibiotics were prescribed for $346(24.2 \%)$ patients for prevention or treatment of secondary bacterial infections.

Symptomatic treatment including anti-inflammatory agents (steroids, IL-6 inhibitorsarilumab) and/or convalescent plasma was recorded for 167 (11.7\%) cases. Anticoagulant therapy (Enoxaparin sodium or Heparin) was given to $15(1 \%)$ patients. Fifty $(3.5 \%)$ patients did not receive any pharmacological treatment.

Information on the use of supportive care and therapy was available in 1054 patients. Of these, 352 patients needed supplemental oxygen via venturi or face masks; 211 patients required non-invasive mechanical ventilation (bi-level positive airway pressure-BiPAP, or continuous positive airway pressure- $\mathrm{CPAP}$ ) and 144 patients needed invasive ventilation, of whom, three were given extracorporeal membrane oxygenation (ECMO).

Information on the status of treatment for HIV while combating COVID-19 was available in 206 cases. The treatment for HIV was maintained for 190 patients, while in 8 patients, it was modified and in the last 8 patients, it was discontinued temporarily.

\subsection{Symptoms of COVID-19}

Results from studies on COVID-19 symptoms experienced by PLHIV throughout their illness are detailed in Supplementary Table S9. The symptoms that were analyzed in the current review and the results for the pooled prevalence of symptoms are as summarized in Table 2. Meta-analysis showed that fever was the most common symptom (79.1\%, 95\% CI 65.8-76.4) followed by dry cough (66.3\%, 95\% CI 58.0-74.7) and dyspnoea (46.0\%, 95\% CI 34.3-57.6). General symptoms such as lethargy and myalgia were also quite frequent (33.6\%, 95\% CI 20.6-46.7; and $28.1 \%$, 95\% CI 18.7-37.5 respectively). Less frequent but widely recognised symptoms were gastrointestinal upsets, anosmia and ageusia, chest discomfort, and mental changes. Overall, the heterogeneity between the pooled studies was relatively high, with $\mathrm{I}^{2}$ greater that $75 \%$ in half the cases (Forest plots are for the symptoms are shown in Supplementary Figure S1-S16). 
Table 2. Pooled prevalence and 95\% confidence interval (CI) of COVID-19 symptoms.

\begin{tabular}{|c|c|c|c|c|c|c|c|c|c|}
\hline Category & Symptoms & $\begin{array}{l}\text { No. of } \\
\text { Studies }\end{array}$ & $\begin{array}{l}\text { No. of } \\
\text { Patients }\end{array}$ & $\begin{array}{l}\text { Total Number of } \\
\text { PLHIV Co-Infected } \\
\text { with COVID-19 }\end{array}$ & $\begin{array}{c}\text { Pooled } \\
\text { Prevalence }\end{array}$ & $95 \%$ CI & $I^{2}$ & $p$-Value & Supplementary \\
\hline & Asymptomatic & 5 & 26 & 196 & 13.2 & $3.6,22.7$ & 75.9 & 0.002 & Figure S1 \\
\hline \multirow{2}{*}{$\begin{array}{l}\text { Constitutional } \\
\text { symptoms }\end{array}$} & Fever & 14 & 614 & 863 & 71.1 & $65.8,76.4$ & 61.8 & 0.001 & Figure S2 \\
\hline & Lethargy & 7 & 245 & 600 & 33.6 & $20.6,46.7$ & 90.9 & $<0.001$ & Figure S3 \\
\hline \multirow{4}{*}{$\begin{array}{l}\text { Respiratory } \\
\text { symptoms }\end{array}$} & Dry cough & 13 & 571 & 832 & 66.3 & $58.0,74.7$ & 84.3 & $<0.001$ & Figure S4 \\
\hline & Dyspnoea & 12 & 405 & 799 & 46.0 & $34.3,57.6$ & 91.1 & $<0.001$ & Figure S5 \\
\hline & Nasal congestion & 5 & 61 & 560 & 8.8 & $4.7,12.8$ & 61.2 & 0.036 & Figure S6 \\
\hline & Sore throat & 7 & 109 & 618 & 16.0 & $11.5,20.5$ & 48.9 & 0.068 & Figure S7 \\
\hline \multirow{3}{*}{$\begin{array}{l}\text { Gastrointestinal } \\
\text { manifestations }\end{array}$} & Abdominal pain & 4 & 18 & 189 & 8.4 & $4.4,12.3$ & $<0.000$ & 0.455 & Figure S8 \\
\hline & Diarrhoea & 10 & 157 & 739 & 19.0 & $14.0,24.0$ & 62.3 & 0.005 & Figure S9 \\
\hline & Nausea & 6 & 104 & 555 & 13.8 & $6.8,20.8$ & 80.2 & $<0.001$ & Figure S10 \\
\hline \multirow{4}{*}{$\begin{array}{l}\text { Neurological } \\
\text { symptoms }\end{array}$} & Ageusia & 5 & 51 & 442 & 9.7 & $4.4,15.0$ & 65.2 & 0.022 & Figure S11 \\
\hline & Anosmia & 9 & 81 & 645 & 11.5 & $6.0,17.0$ & 82.7 & $<0.001$ & Figure S12 \\
\hline & Headache & 10 & 158 & 752 & 17.9 & $11.0,24.8$ & 84.5 & $<0.001$ & Figure S13 \\
\hline & $\begin{array}{c}\text { Mental status } \\
\text { changes/Confusion }\end{array}$ & 3 & 34 & 415 & 8.0 & $5.4,10.6$ & 0.0 & 0.631 & Figure S14 \\
\hline Musculoskeletal & Myalgia & 11 & 259 & 757 & 28.1 & $18.7,37.5$ & 87.2 & $<0.001$ & Figure S15 \\
\hline $\begin{array}{l}\text { Cardiac } \\
\text { symptoms }\end{array}$ & $\begin{array}{l}\text { Chest pain/chest } \\
\text { tightness }\end{array}$ & 4 & 86 & 455 & 18.5 & $14.9,22.0$ & 0 & 0.406 & Figure S16 \\
\hline
\end{tabular}

\subsection{Blood Parameters among PLHIV Co-Infected with COVID-19}

Data on the blood parameters which include inflammatory markers, serum enzymes, serum creatinine and haematological parameters are presented in Table 3. Generally, a fairly high percentage of studies reported elevated baseline values in most of the inflammatory markers (C-reactive protein, fibrinogen, ferritin, and interleukin-6), markers of clotting disorder (D-dimer) and markers of cellular/tissue damage (in particular lactate dehydrogenase). The percentage of cases with elevated biomarkers indicative of inflammation, coagulopathy, and tissue damage was correspondingly high. The results of the liver profile were fairly variable across studies and the effect of COVID-19 on these analytes was inconsistent. The mean serum creatinine level was within the reference limits in over two thirds of the studies. Haemoglobin and leukocytes were normal in most of the patients while lymphocytes were low in about $50 \%$ of cases. 
Table 3. Blood parameters among PLHIV co-infected with COVID-19.

\begin{tabular}{|c|c|c|c|c|c|c|}
\hline Category of Marker & $\begin{array}{l}\text { Blood Parameters } \\
\text { [Reference Limits] }\end{array}$ & $\begin{array}{c}\text { Overall Range of } \\
\text { Mean }\end{array}$ & $\begin{array}{l}\text { Mean: Decreased, } \\
\text { Normal or Elevated }\end{array}$ & $\begin{array}{l}\text { No. of } \\
\text { Studies }\end{array}$ & $\begin{array}{l}\text { No. of } \\
\text { Patients }\end{array}$ & $\begin{array}{l}\text { Range of Mean in } \\
\text { Each Sub-Category }\end{array}$ \\
\hline \multirow{13}{*}{ Inflammation } & \multirow{3}{*}{$\begin{array}{c}\text { C-reactive protein }(\mathrm{mg} / \mathrm{dL}) \\
{[0.3-1.0]}\end{array}$} & \multirow{3}{*}{$0.06-130$} & Low & 1 & 20 & 0.06 \\
\hline & & & Normal & 3 & 5 & $0.46-0.5$ \\
\hline & & & Elevated & 42 & 1015 & $1.13-130$ \\
\hline & \multirow{2}{*}{$\begin{array}{l}\text { Ferritin (ng/mL) } \\
\text { [M: 20-250; F: 10-125] }\end{array}$} & \multirow{2}{*}{$0.484-23,647$} & Normal & 6 & 30 & $0.484-250.3$ \\
\hline & & & Elevated & 19 & 720 & $306.7-23,647$ \\
\hline & \multirow{2}{*}{$\begin{array}{l}\text { Fibrinogen }(\mathrm{mg} / \mathrm{dL}) \\
{[200-400]}\end{array}$} & \multirow{2}{*}{$437-850$} & Normal & Nil & Nil & Nil \\
\hline & & & Elevated & 6 & 109 & $437-850$ \\
\hline & \multirow{2}{*}{$\begin{array}{c}\text { Interleukin-6 (pg/mL]) } \\
\text { [0-16.4 pg/mL; Risk Cut off: 80] }\end{array}$} & \multirow{2}{*}{ 5-139.8 } & Normal & 4 & 40 & $5-12.2$ \\
\hline & & & Elevated & 9 & 237 & $24.7-139.8$ \\
\hline & \multirow{2}{*}{$\begin{array}{l}\text { Procalcitonin }(\mathrm{ng} / \mathrm{mL}) \\
{[<0.15 \text { to } 0.2 \text { mild elevated }]>2} \\
\text { elevated; }>5 \text { to } 10 \text { severe }\end{array}$} & \multirow[t]{2}{*}{$0.03-162$} & $\begin{array}{l}\text { Normal or Mildly } \\
\text { elevated }\end{array}$ & 15 & 285 & $0.03-0.97$ \\
\hline & & & Elevated & 3 & 34 & $2.2-162$ \\
\hline & \multirow{2}{*}{ Troponin (ng/mL) [0-4] } & \multirow{2}{*}{$0.02-25.3$} & Normal & 2 & 22 & $0.02-0.03$ \\
\hline & & & Elevated & 2 & 56 & $0.89-25.3$ \\
\hline \multirow{2}{*}{$\begin{array}{l}\text { Coagulation/fibrinolysis } \\
\text { Severity/prognosis (COVID) }\end{array}$} & \multirow{2}{*}{ D-dimer (ng/mL) } & \multirow{2}{*}{$0.4-8854$} & Normal & 4 & 53 & $0.4-207$ \\
\hline & & & Elevated & 23 & 444 & $333-8854$ \\
\hline \multirow{4}{*}{ Tissue damage } & \multirow{2}{*}{$\begin{array}{l}\text { Lactate dehydrogenase (U/L) } \\
\qquad[140-280]\end{array}$} & \multirow{2}{*}{$169-1200$} & Normal & 8 & 128 & $169-273$ \\
\hline & & & Elevated & 21 & 650 & $312-1200$ \\
\hline & \multirow{2}{*}{$\begin{array}{c}\operatorname{AST}(\mathrm{U} / \mathrm{L}) \\
{[\mathrm{M}:<50 ; \mathrm{F}:<45]}\end{array}$} & \multirow{2}{*}{$24-477$} & Normal & 10 & 514 & $24-48.4$ \\
\hline & & & Elevated & 6 & 107 & $50.5-477$ \\
\hline \multirow{8}{*}{ Liver function } & \multirow{2}{*}{$\begin{array}{l}\text { Albumin }(g / L) \\
{[35-55]}\end{array}$} & \multirow{2}{*}{$0.03-37.7$} & Decreased & 4 & 30 & $0.03-33.2$ \\
\hline & & & Normal & 2 & 22 & $35.7-37.7$ \\
\hline & \multirow{2}{*}{$\begin{array}{l}\mathrm{ALP}(\mathrm{U} / \mathrm{L}) \\
{[40-140]}\end{array}$} & 42-198 & Normal & 7 & 104 & $42-128.4$ \\
\hline & & $4<-190$ & Elevated & 1 & 1 & 198 \\
\hline & $\operatorname{ALT}(\mathrm{U} / \mathrm{L})$ & $11-363$ & Normal & 10 & 550 & $11-32$ \\
\hline & [M: <33; F: <25] & & Elevated & 9 & 591 & $37.2-363$ \\
\hline & AST (U/L) & $24-477$ & Normal & 10 & 514 & $24-48.4$ \\
\hline & [M: $<50 ; \mathrm{F}:<45]$ & $24-4 / 7$ & Elevated & 6 & 107 & $50.5-477$ \\
\hline & Haemoglobin (g/dL) & $36-161$ & Decreased & 4 & 27 & $3.6-11.7$ \\
\hline & [M: 130-170; F: 120-160] & $0.0-10.1$ & Normal & 12 & 212 & $12.2-16.1$ \\
\hline & & & Decreased & 8 & 153 & $0.197-130$ \\
\hline & $\begin{array}{c}\text { Platelets }\left(\times 10^{9} / \mathrm{L}\right) \\
{[150-450]}\end{array}$ & $0.197-276,000$ & Normal & 15 & 149 & $148-278$ \\
\hline & & & Elevated & 2 & 3 & $170 \mathrm{~K}-276 \mathrm{~K}$ \\
\hline & & & Decreased & 9 & 34 & $0.46-3.94$ \\
\hline Haematological status & $\begin{array}{c}\text { Leukocyte }\left(\times 10^{9} / \mathrm{L}\right) \\
{[4.0-11]}\end{array}$ & $0.46-15.1$ & Normal & 31 & 880 & $4.1-10.8$ \\
\hline & & & Elevated & 2 & 6 & $12.9-15.1$ \\
\hline & & & Decreased & 27 & 445 & $0.4-1.44$ \\
\hline & $\begin{array}{c}\text { Lymphocytes }\left(\times 10^{9} / \mathrm{L}\right) \\
{[1.5-4.5]}\end{array}$ & $0.4-26$ & Normal & 10 & 116 & $1.48-2.45$ \\
\hline & & & Elevated & 3 & 341 & $13.1-26$ \\
\hline & Neutrophil $\left(\times 10^{9} / \mathrm{L}\right)$ & 197-103 & Normal & 7 & 143 & $1.97-6.7$ \\
\hline & {$[2.0-7.5]$} & 1. & Elevated & 1 & 1 & 10.3 \\
\hline
\end{tabular}

\subsection{Subgroup Analysis of PLHIV on HAART Compared with Those Who Were Not}

Results of subgroup analysis on selected laboratory findings and clinical features of PLHIV co-infected with COVID-19 who (i) were on HAART and compliant with the treatment $(n=19)$ and (ii) those who were not-reason unspecified $(n=9)$ are shown in Table 4. 
Table 4. Subgroup analysis on characteristics of PLHIV co-infected with COVID-19 adherence to Highly active antiretroviral therapy (HAART).

\begin{tabular}{|c|c|c|c|}
\hline Factors & Characteristics & On HAART $(n=19)$ & Not on HAART $(n=9)$ \\
\hline \multirow{2}{*}{$\begin{array}{l}\text { HIV profile, mean } \\
\text { (range) }\end{array}$} & Average CD4+ & $556.9($ range $=201-1827)$ & $331.8($ range $=10-504)$ \\
\hline & Average Viral load & 24.7 (range = 20-40 RNA copies $/ \mathrm{mL}$ ) & $\begin{array}{c}50,492.5 \text { (range }=20-293,313 \text { RNA } \\
\text { copies } / \mathrm{mL})\end{array}$ \\
\hline \multirow{6}{*}{$\begin{array}{l}\text { Symptoms (Number of } \\
\text { individuals with } \\
\text { symptom) }\end{array}$} & General & $\begin{array}{c}\text { Nausea (4), decreased appetite (1), } \\
\text { malaise (1), lethargy (2) } \\
\text { Fever (15), chills (1), myalgia (3), } \\
\text { headache (3) }\end{array}$ & $\begin{array}{l}\text { Nausea (1), myalgia (2), } \\
\text { Fever (5), chills (2), night sweat (1), } \\
\text { headache (3) }\end{array}$ \\
\hline & Upper respiratory tract & Sore throat (2), nasal congestion (1) & Sore throat (1) \\
\hline & Lower respiratory system & $\begin{array}{l}\text { Dyspnoea }(10 / 19=52.6 \%) \text {, productive } \\
\text { cough }(4), \text { dry cough }(11), \text { tachypnoea }(1)\end{array}$ & $\begin{array}{c}\text { Dyspnoea }(6 / 9=66.7 \%) \text {, productive } \\
\text { cough }(1), \text { dry cough }(5)\end{array}$ \\
\hline & Gastrointestinal tract & Abdominal pain (2), diarrhoea (6) & Abdominal pain (2) \\
\hline & Central nervous system & Mental status changed (1) & Nil \\
\hline & Cardiovascular system & Palpitation (3) & Chest pain (1), palpitation (1) \\
\hline \multirow{6}{*}{$\begin{array}{l}\text { Laboratory markers, } \\
\text { mean (range) }\end{array}$} & C-reactive protein $(\mathrm{mg} / \mathrm{dL})$ & $\begin{array}{c}18.9 \text { range }=0.49-120) ; \text { Elevated }=93.8 \% \\
\text { (Based on } 16 \mathrm{pts})\end{array}$ & $\begin{aligned} 20.9(\text { range }= & 0.25-53.2) ; \text { Elevated }=87.5 \% \\
& (\text { Based on } 8 \text { pts })\end{aligned}$ \\
\hline & D-dimer (ng/mL) & $\begin{array}{c}1343.9(\text { range }=177-6077) ; \text { Elevated }=83.3 \% \\
\text { (Based on } 12 \text { pts })\end{array}$ & $\begin{array}{c}433.5(\text { range }=37-790) ; \text { Elevated }=75 \% \\
(\text { Based on } 4 \text { pts })\end{array}$ \\
\hline & Ferritin (ng/mL) & $\begin{array}{c}880.8 \text { (range }=46-5937) ; \text { Elevated }=66.7 \% \\
\text { (Based on } 12 \text { pts) }\end{array}$ & $\begin{array}{c}371.5 \text { range }=0.5-1010) ; \text { Elevated }=80.0 \% \\
(\text { Based on } 5 \mathrm{pts})\end{array}$ \\
\hline & Fibrinogen $(\mathrm{mg} / \mathrm{dL})$ & $\begin{array}{c}455.8 \text { (range }=182-624) ; \text { Elevated }=75 \% \\
\text { (Based on } 4 \text { pts })\end{array}$ & $\begin{array}{c}450.5 \text { (range }=437-464) ; \text { Elevated }=100 \% \\
\text { (Based on } 2 \text { pts })\end{array}$ \\
\hline & Interleukin-6 (pg/mL) & $\begin{array}{c}60.2(\text { range }=9.9-213) ; \text { Elevated }=57.1 \% \\
\text { (Based on } 7 \text { pts })\end{array}$ & $\begin{array}{c}163.5 \text { (range }=75.9-251) ; \text { Elevated }=100 \% \\
\text { (Based on } 2 \text { pts })\end{array}$ \\
\hline & $\begin{array}{l}\text { Lactate dehydrogenase } \\
\qquad(\mathrm{U} / \mathrm{L})\end{array}$ & $\begin{array}{c}303.4 \text { (range }=162-467) ; \text { Elevated }=60 \% \\
(\text { Based on } 5 \text { pts })\end{array}$ & $\begin{array}{c}616.3 \text { range }=308-1200) ; \text { Elevated }=100 \% \\
\text { (Based on } 4 \text { pts })\end{array}$ \\
\hline Chest Imaging Studies & Abnormal findings & $18(94.7)$ & $6(66.7)$ \\
\hline \multirow{3}{*}{ Disease severity } & Hospitalization & $19(100)$ & $9(100)$ \\
\hline & ICU admission & $10(52.6)$ & $4(44.4)$ \\
\hline & Mortality & $6(31.6)$ & $3(33.3)$ \\
\hline
\end{tabular}

Those who were on HAART had a mean CD4 count of 556.9 (range $=201-1827$ ) while those who were not had a mean count of 331.8 (range $=10-504$ ). As expected, the mean viral load among those on HAART was significantly lower with a mean of 24.7 (range $=20-40$ RNA copies $/ \mathrm{mL}$ ) compared to who were not $($ mean $=50,493 ;$ range $=20$ $293,313 / \mathrm{mL}$ ). These results support a more serious HIV infection in those not on HAART, regardless of the reason, and by inference a greater degree of cellular immune-deficiency, which is supported by the HIV disease classification based on clinical criteria [105].

However, the clinical presentation of COVID was quite similar in both groups of HIV patients; likewise, disease severity as judged by the number of ICU admissions. Similarly, the acute inflammatory markers, D-dimer and lactic dehydrogenase, were elevated in both groups of HIV individuals to a similar extent. The apparent higher levels of D-dimer and ferritin among those on HAART and higher levels of IL-6 and lactic dehydrogenase among those not on HAART are of no consequence in view of the very small number of cases with these results.

\section{Discussion}

The purpose of this study is to systematically review and conduct meta-analysis using data from current studies reporting on epidemiological characteristics and outcomes, and the clinical course in PLHIV co-infected with COVID-19. A limited comparison of clinical features, laboratory findings, and disease severity (based on hospitalization, admission to ICU and death) between those on HAART and those who were not was also included. 


\subsection{Epidemiology, Comorbidities, and Outcomes of COVID-19 in PLHIV}

In the current review, the overall PIP of COVID-19 among PLHIV from meta-analysis was $0.9 \%\left(95 \%\right.$ CI $\left.0.6 \%, 1.1 \% ; I^{2}=98.5 \%\right)$. To address the high degree of heterogeneity across the seven cohort studies used for the estimation of the overall PIP of COVID-19, leave-one-out meta-analysis was performed. The results demonstrated that each of these studies had substantial effect ( $p$-value $<0.001$ ) on the overall estimate of the rest of the studies (PIP varying from 0.7 to 1.0). (Supplementary Table S10: Summary of estimates and its 95\% CI from leave-one-out Meta-analysis; Supplementary Figure S17: Leaveone-out Meta-analysis forest plot). The heterogeneity between these studies is further demonstrated by the forest plot (Figure 2) and by the $\mathrm{I}^{2}$ result and the corresponding $p$-value. The incidence proportion between studies ranged from 0.3\% (Del Amo et al.) [30] to $1.9 \%$ (Vizcarra et al.) [88]. Methodological variations such as sampling methods, study design and study size (range: 902 to $>530,000$ PLHIV) are noted as possible contributary factors for this finding.

Overall, $28.4 \%$ of PLHIV affected by COVID-19 were admitted to hospital. The infection was classified as severe-critical in $2.5 \%$ of patients and $3.5 \%$ of patients were admitted to the intensive care unit. The mortality rate was $5.3 \%$. Severe or critical COVID-19 patients were older (54.8 years \pm 13.2 ) compared to non-severe/critical cases ( 46.7 years \pm 11.8 ). Further, among the severe/critical cases, the frequency of comorbidities was much higher compared to the non-severe/critical cases. For example, hypertension and diabetes were present in $25 \%$ and $10 \%$ in the former compared to $13.9 \%$ and $7.6 \%$, respectively, in the latter group. These results are consistent with those reported in previous reviews in which older age and the presence of comorbid conditions, in particular hypertension or diabetes, were associated with severe COVID-19 [106].

The most common comorbidity among all PLHIV suffering from COVID-19 was hypertension $(23.9 \%)$ followed by diabetes $(12.2 \%)$. Previous reviews have similarly reported a high prevalence of hypertension (14.3\% to $27.4 \%$ ) and diabetes (7.7\% to $17.4 \%)$ in the general population infected with COVID-19 [5,107-110]. In another review on the prevalence of comorbidities among fatal COVID-19 cases, hypertension was also the most common $(38.6 \%)$ followed in decreasing frequency by diabetes $(22.2 \%)$, chronic cardiovascular disease $(17.5 \%)$, and cerebrovascular disease (15.6\%) [111]. Pranata et al. reported that hypertension was associated with severe COVID-19 (risk ratio 2.04), intensive care unit admissions (risk ratio 2.11), and mortality (risk ratio 2.11) [112]. These observations clearly demonstrate that hypertension is the most prevalent comorbidity among people with COVID-19 and is also a risk factor for adverse outcomes.

This is not surprising as hypertension is one of the most common chronic diseases with a global prevalence of approximately $31.1 \%$ in the year 2010 [113]. Another possible explanation for hypertension being a risk factor for severe outcome and mortality could be that hypertensive patients have increased angiotensin-converting enzyme 2 expression [114] and this increases the risk of the patient to severe COVID-19 as more of the SARS-CoV-2 virus can bind with the increased number of ACE2 receptors in the lungs to enter cells [115]. A point of note in this regard is that hypertensive patients are often treated with angiotensin-converting enzyme inhibitors and angiotensin receptor blockers. However, some studies have shown that these pharmacological treatments increase angiotensin-converting enzyme 2 expressions [116,117], thereby facilitating the entry of SARS-CoV-2 to cause severe outcomes and mortality [118].

It is noteworthy that lifestyle related disorders appear to be quite common, in particular obesity and dyslipidaemia, followed by smoking and illicit drug intake, the last not unexpected, being a risk factor for HIV infection.

\subsection{Chest Imaging Findings in PLHIV Co-Infected with COVID-19}

Chest imaging examinations are useful in monitoring hospitalised patients particularly those with moderate to severe symptoms and those at risk of progression, as well as for the evaluation of complications. Further, they can be useful for medical triage of patients with 
moderate or severe symptoms in settings with limited resources for nucleic acid-based diagnosis, and act as prompts for clinicians to be cautious with patients who might have COVID-19 despite repeated negative nucleic acid-based test results. It is noted, however, that chest imaging findings are quite varied and dependent on the stage and severity of the illness, and underlying comorbidities [119,120].

In this review, the more common abnormalities of clinical importance reported were ground-glass opacities (GGO), consolidation, and reticulation. Of these, GGO, widely recognised as an early and typical feature, was the most frequent abnormality observed, being found in $72.2 \%$ of patients with mild-to-moderate disease, and $68.8 \%$ of patients with severe-critical disease. The distribution of ground glass infiltrations was predominantly bilateral, which is similar to that described in several reviews [121-124].

Apart from GGO, consolidation, and reticulation, the wide variety of other imaging findings that were reported by the various studies in this series was similar to that reported, albeit at varying frequencies, in COVID-19 patients in general. Finally, in most patients with either bilateral $(75 \%)$ or unilateral pneumonia $(25 \%)$, chest imaging manifestations were similar regardless of COVID-19 severity.

\subsection{Clinical Features in PLHIV Co-Infected with COVID-19}

The most common symptom in PLHIV co-infected with COVID-19 was fever (71.1\%), a finding broadly consistent with previous reviews which reported an overall frequency ranging from $85.6 \%$ to $91.3 \%[5,107,122]$. In a separate review on COVID-19 in cancer patients [115], fever was found to be present in $85.4 \%$ of cases. Less frequently reported symptoms include headache $(17.9 \%)$, altered mental status $(8.0 \%)$, anosmia $(11.5 \%)$, and ageusia $(9.7 \%)$. The prevalence of these relatively uncommon symptoms is highly variable across previous reviews [125-127] giving rise to concerns. For example, the reported frequency of impaired smell sensation varied between $8.3 \%$ and 59.9\% and that for impaired taste sensation varied between $6.7 \%$ and $57.5 \%$. The reported frequency of impaired consciousness was between 1.6 and $5.0 \%$. While headache may be multifactorial in its aetiopathogenesis, anosmia, ageusia, and altered mental status are clearly neurological in origin, and thus of clinical interest and relevance.

SARS-COVID-2 has been known to exhibit neurotrophic properties and can directly infect the nervous system [128]. The ACE2 receptors which are the cellular entrance for this virus have been found to be abundant not only on epithelial cells of the tongue [129] and lungs [130] but also on glial cells and neurons. Therefore, the virus could possibly gain entry into the central nervous system via a neuronal pathway or neuronal transport, thereby causing damage to nerve functions [131]. Chilvers et al. reported that the virus is able to disrupt nasal epithelium and is released on the apical and basolateral side of epithelial cells to reach the central nervous system via the bloodstream [132]. However, the exact process by which olfactory and gustatory dysfunctions and damage occur remains to be elucidated. Based on the neurotrophic potential of SARS-CoV-2, some have suggested the possibility that these neurological symptoms might persist even after patients have recovered from COVID-19 [133], but to-date, there is still no evidence to support this assumption.

\subsection{Blood Parameters in PLHIV Co-Infected with COVID-19}

Most studies $[111,134]$ have demonstrated an abnormal elevation of various inflammation markers in COVID-19 patients, which could be indicative of a hyper-inflammatory state and poor disease prognosis.

The changes in white blood cell counts in COVID-19 are variable depending on the clinical phase of the illness. Generally, lymphopenia, which is reported in many studies, is evident with the onset of symptomatic disease during which the systematic inflammatory response or even the "Cytokine storm" may occur in more severe cases. This observation is partially ascribed to the presence of ACE2 receptors on the surface of lymphocytes allowing direct injury by the SARS-CoV-2 virus. Alternatively, the release of inflammatory markers including interleukins, granulocyte colony stimulating factor (GCSF) and tumor 
necrosis factor alpha (TNF- $\alpha$ ) may induce apoptosis of the cells. Hence, the observed association between lymphopenia and severity or poor prognosis of the illness. Indeed, in the present review, lymphopenia was present in an average of $42.6 \%$ of symptomatic individuals, compared to $20 \%$ of asymptomatic individuals. Other findings of note are increased neutrophils in $37.5 \%$ and decreased platelets in $39.1 \%$ of symptomatic cases. However, sequential changes in blood counts were not documented in most studies on PLHIV with COVID-19. In this review, we found only three studies which reported changes in full blood counts $[55,81,102]$; however, all three reported cell counts within the normal range on admission and throughout hospitalization. While this may be the case, it is not unreasonable to expect that PLHIV will demonstrate a blood cell profile largely similar to that of non-HIV infected people as a response to the infection.

\subsection{Comparison between PLHIV with COVID-19 on HAART and Those Who Were Not}

Immunocompromised individuals are expected to be more highly susceptible to severe COVID-19 [135]. However, this general assumption was not borne out by the results of our study. In our subgroup comparison, PLHIV who were on the HAART regimen had higher CD4 cell counts, and lower HIV viral loads compared to those who were not, as expected. Logically, the former group should have better cellular immunity than the latter. However, subgroup analysis did not demonstrate any relevant difference with respect to the clinical features, laboratory findings, and severity of illness. It is acknowledged, however, that the number of subjects with data for this sub-group analysis was too small to provide any definite conclusions.

\subsection{What Are the Impacts of COVID-19 on Health Care for People with HIV?}

The rapid spread and exponential increase in the number of COVID-19 infections globally has strained healthcare systems due to the diversion of resources from less urgent services to COVID-19 control and management [136]. This would be expected to impact negatively the delivery of care for other illnesses. With specific reference to HIV infection, this could result in disruption of the broad spectrum of care including the initiation and maintenance of ART [137-139]. In the broader perspective, the UNAIDS' first 90-90-90 target for 2020 will be challenged [140]. Research and development funding and activity have also been diverted to or focussed on COVID-19 [141]. However, it should be noted that measures have been taken to minimise these negative effects through streamlining of processes and the use of the internet [142].

\subsection{Is COVID-19 Vaccine Safe for PLHIV?}

Currently, several types of vaccines including mRNA, viral vector, inactivated whole virus, and protein subunit vaccines are in phase 3 clinical trials, some of which have been approved for emergency use to protect against serious complications of the infection. Of these different vaccines, data on the trials that included PLHIV are relatively limited. These include trials that involved the Pfizer/BioNTech (BNT162b2) mRNA vaccine [143], the Moderna mRNA (mRNA-1273) vaccine [144], the Oxford-AstraZeneca viral vector vaccine (AZD1222) [145], and the Janssen viral vector vaccine (Ad26.COV2.S) [146]. The reported efficacy in terms of preventing symptomatic illness, and moderate as well as severe/critical illnesses ranged from $66 \%$ to $95 \%$ [145-148]. The data on safety of the vaccines are also limited due to the short time frame since vaccination studies commenced. Of note is that the numbers of PLHIV included in these studies were relatively small and data on their response to vaccination were not separately reported. This underlies the need for more studies to address this question. It is also noted that the emergence of new strains of the SARS-CoV-2 virus will further complicate the picture.

\subsection{Does COVID-19 Affect Patients with Long-Term HIV More?}

Data on the severity of COVID illness among PLHIV in relation to the duration of HIV infection was available in a small number of cases. Based on arbitrary definition 
for long-term HIV (diagnosed with HIV for at least 8 years or longer), 71 cases were considered as long-term HIV patients and 40 as short term. Briefly, 41 (57.7\%) long-term HIV infected cases had severe/critical COVID illness compared to 20 (50.0\%) of those with short-term HIV infection while the admission rate to ICU was $26.7 \%$ and $7.5 \%$, respectively. From the foregoing data, it would appear that long-term HIV infection is associated with more severe illness; however, the significance of these figures needs to be established with further studies.

\subsection{Strengths and Limitations}

This review contributes to existing knowledge of COVID-19 infection in PLHIV by providing a comprehensive investigation of epidemiological characteristics, clinical signs and symptoms, blood parameters, and clinical outcomes based on a large sample size of more than 6000 PLHIV co-infected with COVID-19. Being limited by the unavailability of some data and the small sample size in some studies, we were unable to conduct a meta-analysis for all parameters. In the subgroup comparison (patients on HAART versus patients not on HAART), there were few patients in both subgroups (19 and 9 cases, respectively). As for the prevalence of asymptomatic patients, it was unclear how these patients were detected. This could be dependent on the community and environment in which the PLHIV lived and the intensity of the detection effort by the health authorities. As such, there could be a considerable degree of detection bias giving rise to unreliable estimates of the proportion of asymptomatic cases in the study.

\section{Conclusions}

This study has shown a COVID-19 pooled incidence proportion of $0.9 \%$ (95\% CI $0.6 \%, 1.1 \%$ ) among PLHIV and 5.3\% of mortality for PLHIV co-infected with COVID-19. Hypertension and fever were the most common comorbidity and symptom, respectively. Most inflammation markers were elevated on admission. As the COVID-19 pandemic is still rapidly evolving, further studies are needed to corroborate the results of this review. Considerably more data will need to be analyzed particularly for the comparison of PLHIV with COVID-19 on HAART and those who are not.

Supplementary Materials: The following are available online at https: / www.mdpi.com/1660-4 601/18/7/3554/s1, Table S1: PRISMA checklist, Table S2: Search terms used for final search on 20 September 2020, Table S3: Quality appraisal checklist for case series and case report, Table S4: COVID19 Disease Severity as defined by World Health Organization, Table S5: Information on comorbidities and lifestyle-related disorders among patients with HIV+COVID-19 in the study, Table S6: HIV profile of patients with HIV+Covid-19, Table S7: Laboratory results, chest imaging findings and vital signs among HIV+Covid-19, Table S8: Pharmacological treatment and supportive care given to HIV+COVID19 patients, Table S9: Findings from studies regarding symptoms experienced by patients throughout clinical course, Table S10: Summary of estimate and its 95\% CI for Pooled Incidence proportion of COVID-19 among PLHIV based on data accumulated from cohort studies using leave-one-out Meta-analysis forest plot. Figure S1: Forest plot for pooled prevalence of asymptomatic PLHIV with COVID-19, Figure S2: orest plot for pooled prevalence of fever in PLHIV with COVID-19, Figure S3: Forest plot for pooled prevalence of lethargy in PLHIV with COVID-19, Figure S4: Forest plot for pooled prevalence of dry cough in PLHIV with COVID-19, Figure S5: Forest plot for pooled prevalence of dyspnoea in PLHIV with COVID-19, Figure S6: Forest plot for pooled prevalence of nasal congestion in PLHIV with COVID-19, Figure S7: Forest plot for pooled prevalence of sore throat in PLHIV with COVID-19, Figure S8: Forest plot for pooled prevalence of abdominal pain in PLHIV with COVID-19, Figure S9: Forest plot for pooled prevalence of diarrhoea in PLHIV with COVID-19, Figure S10: Forest plot for pooled prevalence of nausea in PLHIV with COVID-19, Figure S11: Forest plot for pooled prevalence of ageusia in PLHIV with COVID-19, Figure S12: Forest plot for pooled prevalence of anosmia in PLHIV with COVID-19, Figure S13: Forest plot for pooled prevalence of headache in PLHIV with COVID-19, Figure S14: Forest plot for pooled prevalence of altered mental status/confusion in PLHIV with COVID-19, Figure S15: Forest plot for pooled prevalence of myalgia in PLHIV with COVID-19, Figure S16: Forest plot for pooled prevalence of 
chest pain/chest tightness in PLHIV with COVID-19, Figure S17: Leave-one-out Meta-analysis forest plot for pooled incidence proportion of COVID-19 among PLHIV based on data accumulated from cohort studies.

Author Contributions: Conceptualization, K.W.L., S.F.Y., Y.F.N., and M.S.L.; Formal analysis, K.W.L., S.F.Y., and M.S.L.; Investigation, K.W.L., S.F.Y., Y.F.N., and M.S.L.; Methodology, K.W.L. and Y.F.N.; Project administration, K.W.L. and S.F.Y.; Software, K.W.L.; Supervision, S.F.Y. and M.S.L.; Validation, S.F.Y., Y.F.N., and M.S.L.; Visualization, K.W.L.; Writing-original draft, K.W.L., S.F.Y., Y.F.N., and M.S.L.; Writing—review and editing, K.W.L., S.F.Y., Y.F.N., and M.S.L. All authors have read and agreed to the published version of the manuscript.

Funding: This research did not receive any specific grant from funding agencies in the public, commercial, or not-for-profit sectors.

Institutional Review Board Statement: Not applicable.

Informed Consent Statement: Not applicable.

Conflicts of Interest: All other authors declare no competing interests.

$\begin{array}{ll}\text { Abbreviations } \\ \text { ART } & \text { Antiretrovirus therapy } \\ \text { HAART } & \text { Highly active antiretroviral therapy } \\ \text { PIP } & \text { Pooled incidence proportion } \\ \text { PLHIV } & \text { People living with human immunodeficiency virus }\end{array}$

\section{References}

1. Sohrabi, C.; Alsafi, Z.; O’Neill, N.; Khan, M.; Kerwan, A.; Al-Jabir, A.; Iosifidis, C.; Agha, R. World Health Organization declares global emergency: A review of the 2019 novel coronavirus (COVID-19). Int. J. Surg. 2020, 76, 71-76.

2. Chen, N.; Zhou, M.; Dong, X.; Qu, J.; Gong, F.; Han, Y.; Qiu, Y.; Wang, J.; Liu, Y.; Wei, Y. Epidemiological and clinical characteristics of 99 cases of 2019 novel coronavirus pneumonia in Wuhan, China: A descriptive study. Lancet 2020, 395, 507-513.

3. Lippi, G.; Plebani, M. Laboratory abnormalities in patients with COVID-2019 infection. Clin. Chem. Lab. Med. 2020, 58, 1131-1134.

4. Liu, K.; Chen, Y.; Lin, R.; Han, K. Clinical features of COVID-19 in elderly patients: A comparison with young and middle-aged patients. J. Infect. 2020, 80, e14-e18.

5. Yang, J.; Zheng, Y.; Gou, X.; Pu, K.; Chen, Z.; Guo, Q.; Ji, R.; Wang, H.; Wang, Y.; Zhou, Y. Prevalence of comorbidities in the novel Wuhan coronavirus (COVID-19) infection: A systematic review and meta-analysis. Int. J. Infect. Dis. 2020, 94, 91-95.

6. Wang, D.; Hu, B.; Hu, C.; Zhu, F.; Liu, X.; Zhang, J.; Wang, B.; Xiang, H.; Cheng, Z.; Xiong, Y. Clinical characteristics of 138 hospitalized patients with 2019 novel coronavirus-infected pneumonia in Wuhan, China. JAMA 2020, 323, 1061-1069.

7. Zuin, M.; Rigatelli, G.; Zuliani, G.; Rigatelli, A.; Mazza, A.; Roncon, L. Arterial hypertension and risk of death in patients with COVID-19 infection: Systematic review and meta-analysis. J. Infect. 2020, 81, e84-e86.

8. Wu, Z.-h.; Tang, Y.; Cheng, Q. Diabetes increases the mortality of patients with COVID-19: A meta-analysis. Acta Diabetol. 2020, 1-6. [CrossRef]

9. Alqahtani, J.S.; Oyelade, T.; Aldhahir, A.M.; Alghamdi, S.M.; Almehmadi, M.; Alqahtani, A.S.; Quaderi, S.; Mandal, S.; Hurst, J.R. Prevalence, severity and mortality associated with COPD and smoking in patients with COVID-19: A rapid systematic review and meta-analysis. PLoS ONE 2020, 15, e0233147.

10. Li, X.; Guan, B.; Su, T.; Liu, W.; Chen, M.; Waleed, K.B.; Guan, X.; Gary, T.; Zhu, Z. Impact of cardiovascular disease and cardiac injury on in-hospital mortality in patients with COVID-19: A systematic review and meta-analysis. Heart 2020, 106, $1142-1147$.

11. Mirzaei, H.; McFarland, W.; Karamouzian, M.; Sharifi, H. COVID-19 among people living with HIV: A systematic review. AIDS Behav. 2020, 25, 1-8.

12. Cooper, T.J.; Woodward, B.; Alom, S.; Harky, A. Coronavirus disease 2019 (COVID-19) outcomes in HIV/AIDS patients: A systematic review. HIV Med. 2020, 21, 567-577.

13. Guo, W.; Weng, H.; Bai, H.; Liu, J.; Wei, X.; Zhou, K.; Sande, A. Quick community survey on the impact of COVID-19 outbreak for the healthcare of people living with HIV. Zhonghua Liu Xing Bing Xue Za Zhi 2020, 41, 663-667.

14. Marini, J.J.; Gattinoni, L. Management of COVID-19 respiratory distress. JAMA 2020, 323, 2329-2330.

15. Li, T.; Lu, H.; Zhang, W. Clinical observation and management of COVID-19 patients. Emerg. Microbes Infect. $2020,9,687-690$.

16. Li, L.; Huang, T.; Wang, Y.; Wang, Z.; Liang, Y.; Huang, T.; Zhang, H.; Sun, W.; Wang, Y. COVID-19 patients' clinical characteristics, discharge rate, and fatality rate of meta-analysis. J. Med. Virol. 2020, 92, 577-583.

17. Moher, D.; Shamseer, L.; Clarke, M.; Ghersi, D.; Liberati, A.; Petticrew, M.; Shekelle, P.; Stewart, L.A. Preferred reporting items for systematic review and meta-analysis protocols (PRISMA-P) 2015 statement. Syst. Rev. 2015, 4, 1. 
18. Vandenbroucke, J.P.; Von Elm, E.; Altman, D.G.; Gøtzsche, P.C.; Mulrow, C.D.; Pocock, S.J.; Poole, C.; Schlesselman, J.J.; Egger, M.; Initiative, S. Strengthening the Reporting of Observational Studies in Epidemiology (STROBE): Explanation and elaboration. PLoS Med. 2007, 4, e297.

19. Economics, I.o.H. Quality Appraisal of Case Series Studies Checklist; Institute of Health Economics: Edmonton, AB, Canada, 2014.

20. WHO. HIV/AIDS Clinical Staging, HIV/Aids Case Definitions and Use of HIV Rapid Tests for Diagnosis and Surveillance; WHO Regional Office for South-East Asia: New Delhi, India, 2005.

21. WHO. Clinical Management of Covid-19-Interim Guidance. Available online: https://www.who.int/publications/i/item/ clinical-management-of-covid-19 (accessed on 29 June 2020).

22. Wallace, B.C.; Dahabreh, I.J.; Trikalinos, T.A.; Lau, J.; Trow, P.; Schmid, C.H. Closing the gap between methodologists and end-users: R as a computational back-end. J. Stat. Softw. 2012, 49, 1-15.

23. Inciarte, A.; Gonzalez-Cordon, A.; Rojas, J.; Torres, B.; de Lazzari, E.; de la Mora, L.; Martinez-Rebollar, M.; Laguno, M.; Callau, P.; Gonzalez-Navarro, A.; et al. Clinical characteristics, risk factors, and incidence of symptomatic coronavirus disease 2019 in a large cohort of adults living with HIV: A single-center, prospective observational study. AIDS 2020, 34, 1775-1780. [CrossRef]

24. Maggiolo, F.; Zoboli, F.; Arosio, M.; Valenti, D.; Guarneri, D.; Sangiorgio, L.; Ripamonti, D.; Callegaro, A. SARS-CoV-2 infection in persons living with HIV: A single center prospective cohort. J. Med Virol. 2020, 93, 1145-1149.

25. Vizcarra, P.; Pérez-Elías, M.J.; Quereda, C.; Moreno, A.; Vivancos, M.J.; Dronda, F.; Casado, J.L. Description of COVID-19 in HIV-infected individuals: A single-centre, prospective cohort. Lancet HIV 2020, 7, e554-e564. [CrossRef]

26. Adachi, E.; Saito, M.; Ikeuchi, K.; Hoshina, T.; Yotsuyanagi, H. Cases of coronavirus disease-2019 in HIV-infected transgender women. AIDS 2020, 34, 1435-1436. [CrossRef]

27. Altuntas Aydin, O.; Kumbasar Karaosmanoglu, H.; Kart Yasar, K. HIV/SARS-CoV-2 co-infected patients in Istanbul, Turkey. J. Med. Virol. 2020, 92, 2288-2290.

28. Baluku, J.B.; Mwebaza, S.; Ingabire, G.; Nsereko, C.; Muwanga, M. HIV and SARS-CoV-2 co-infection: A case report from Uganda. J. Med. Virol. 2020, 92, 2351-2353.

29. Benkovic, S.; Kim, M.; Sin, E. 4 Cases: HIV and SARS-CoV-2 Co-infection in patients from Long Island, New York. J. Med. Virol. 2020, 92, 2238-2240.

30. Blanco, J.L.; Ambrosioni, J.; Garcia, F.; Martínez, E.; Soriano, A.; Mallolas, J.; Miro, J.M. COVID-19 in patients with HIV: Clinical case series. Lancet HIV 2020, 7, e314-e316. [CrossRef]

31. Boulle, A.; Davies, M.-A.; Hussey, H.; Ismail, M.; Morden, E.; Vundle, Z.; Zweigenthal, V.; Mahomed, H.; Paleker, M.; Pienaar, D. Risk factors for COVID-19 death in a population cohort study from the Western Cape Province, South Africa. Clin. Infect. Dis. Off. Publ. Infect. Dis. Soc. Am. 2020. [CrossRef]

32. Byrd, K.M.; Beckwith, C.G.; Garland, J.M.; Johnson, J.E.; Aung, S.; Cu-Uvin, S.; Farmakiotis, D.; Flanigan, T.; Gillani, F.S.; Macias-Gil, R.; et al. SARS-CoV-2 and HIV coinfection: Clinical experience from Rhode Island, United States. J. Int. AIDS Soc. 2020, 23, e25573. [CrossRef]

33. Calza, L.; Bon, I.; Borderi, M.; Colangeli, V.; Viale, P. No Significant Effect of COVID-19 on Immunological and Virological Parameters in Patients With HIV-1 Infection. J. Acquir. Immune Defic. Syndr. 2020, 85, e6-e8.

34. Calza, L.; Bon, I.; Tadolini, M.; Borderi, M.; Colangeli, V.; Badia, L.; Verucchi, G.; Rossini, G.; Vocale, C.; Gaibani, P. COVID-19 in patients with HIV-1 infection: A single-centre experience in northern Italy. Infection 2020, 1-5. [CrossRef]

35. Chen, J.; Cheng, X.; Wang, R.; Zeng, X. Computed Tomography Imaging of an HIV-infected Patient with Coronavirus Disease 2019 (COVID-19). J. Med. Virol. 2020. [CrossRef]

36. Chiappe Gonzalez, A.J.; Montenegro-Idrogo, J.J.; Vargas Vadillo, A.R.; Slee Torres, M.; Vargas Matos, I.; Resurrección Delgado, C.P. Hospital-acquired SARS-CoV-2 pneumonia in a person living with HIV. Int. J. STD AIDS 2020. [CrossRef]

37. Childs, K.; Post, F.A.; Norcross, C.; Ottaway, Z.; Hamlyn, E.; Quinn, K.; Juniper, T.; Taylor, C. Hospitalized Patients With COVID-19 and Human Immunodeficiency Virus: A Case Series. Clin. Infect. Dis. 2020, 71, 2021-2022.

38. Cipolat, M.M.; Sprinz, E. COVID-19 pneumonia in an HIV-positive woman on antiretroviral therapy and undetectable viral load in Porto Alegre, Brazil. Braz. J. Infect. Dis. 2020, 24, 455-457.

39. Coleman, H.; Snell, L.B.; Simons, R.; Douthwaite, S.T.; Lee, M.J. Coronavirus disease 2019 and Pneumocystis jirovecii pneumonia: A diagnostic dilemma in HIV. Aids 2020, 34, 1258-1260.

40. Collins, L.F.; Moran, C.A.; Oliver, N.T.; Moanna, A.; Lahiri, C.D.; Colasanti, J.A.; Kelley, C.F.; Nguyen, M.L.; Marconi, V.C.; Armstrong, W.S.; et al. Clinical characteristics, comorbidities and outcomes among persons with HIV hospitalized with coronavirus disease 2019 in Atlanta, Georgia. AIDS 2020, 34, 1789-1794. [CrossRef]

41. Dandachi, D.; Geiger, G.; Montgomery, M.W.; Karmen-Tuohy, S.; Golzy, M.; Antar, A.A.; Llibre, J.M.; Camazine, M.; Santiago, D.D.; Carlucci, P.M. Characteristics, Comorbidities, and Outcomes in a Multicenter Registry of Patients with HIV and Coronavirus Disease-19. Clin. Infect. Dis. 2020. [CrossRef]

42. Del Amo, J.; Polo, R.; Moreno, S.; Díaz, A.; Martínez, E.; Arribas, J.R.; Jarrín, I.; Hernán, M.A. Incidence and severity of COVID-19 in HIV-positive persons receiving antiretroviral therapy: A cohort study. Ann. Intern. Med. 2020, 173, 536-541.

43. Di Biagio, A.; Taramasso, L.; Dentone, C.; Vena, A.; Giacobbe, D.R.; De Maria, A.; Mikulska, M.; Bassetti, M. Is a step-down antiretroviral therapy necessary to fight severe acute respiratory syndrome coronavirus 2 in HIV-infected patients? AIDS 2020, 34, 1865-1867. [CrossRef] 
44. Di Biagio, A.; Ricci, E.; Calza, L.; Squillace, N.; Menzaghi, B.; Rusconi, S.; Orofino, G.; Bargiacchi, O.; Molteni, C.; Valsecchi, L. Factors associated with hospital admission for COVID-19 in HIV patients. AIDS 2020, 34, 1983-1985.

45. Di Giambenedetto, S.; Del Giacomo, P.; Ciccullo, A.; Porfidia, A.; De Matteis, G.; Cianci, R.; De Vito, F.; Dusina, A.; Borghetti, A.; Tumbarello, M. SARS-CoV-2 infection in a highly experienced person living with HIV. AIDS 2020, 34, 1257-1258.

46. d'Ettorre, G.; Recchia, G.; Ridolfi, M.; Siccardi, G.; Pinacchio, C.; Innocenti, G.P.; Santinelli, L.; Frasca, F.; Bitossi, C.; Ceccarelli, G. Analysis of type I IFN response and T cell activation in severe COVID-19/HIV-1 coinfection: A case report. Medicine 2020, 99, e21803.

47. Elhadi, M.; Momen, A.; Abdulhadi, O.; Msherghi, A. Multi-organ failure after acute kidney injury in patient with HIV and COVID-19. New Microbes New Infect. 2020, 37, 100742.

48. Etienne, N.; Karmochkine, M.; Slama, L.; Pavie, J.; Batisse, D.; Usubillaga, R.; Letembet, V.-A.; Brazille, P.; Canouï, E.; Slama, D.; et al. HIV infection and COVID-19: Risk factors for severe disease. AIDS 2020, 34, 1771-1774. [CrossRef]

49. Farinacci, D.; Ciccullo, A.; Borghetti, A.; Visconti, E.; Tamburrini, E.; Izzi, I.M.; Cauda, R.; Di Giambenedetto, S.; Pallavicini, F. People Living with HIV in the COVID-19 Era: A Case Report. AIDS Res. Hum. Retrovir. 2020. [CrossRef]

50. Gadelha Farias, L.A.B.; Gomes Moreira, A.L.; Austregésilo Corrêa, E.; Landim de Oliveira Lima, C.A.; Lopes, I.M.P.; de Holanda, P.E.L.; Nunes, F.R.; Pires Neto, R.d.J. Case Report: Coronavirus Disease and Pulmonary Tuberculosis in Patients with Human Immunodeficiency Virus: Report of Two Cases. Am. J. Trop. Med. Hyg. 2020, 103, 103-1593.

51. Geretti, A.M.; Stockdale, A.J.; Kelly, S.H.; Cevik, M.; Collins, S.; Waters, L.; Villa, G.; Docherty, A.; Harrison, E.M.; Turtle, L. Outcomes of COVID-19 related hospitalisation among people with HIV in the ISARIC WHO Clinical Characterisation Protocol UK Protocol: Prospective observational study. SSRN Electron. J. 2020. [CrossRef]

52. Gervasoni, C.; Meraviglia, P.; Riva, A.; Giacomelli, A.; Oreni, L.; Minisci, D.; Atzori, C.; Ridolfo, A.; Cattaneo, D. Clinical features and outcomes of HIV patients with coronavirus disease 2019. Clin. Infect. Dis. 2020, 71, 2276-2278.

53. Gudipati, S.; Brar, I.; Murray, S.; McKinnon, J.E.; Yared, N.; Markowitz, N. Descriptive Analysis of Patients Living With HIV Affected by COVID-19. JAIDS J. Acquir. Immune Defic. Syndr. 2020, 85, 123-126.

54. Guo, W.; Ming, F.; Feng, Y.; Zhang, Q.; Mo, P.; Liu, L.; Gao, M.; Tang, W.; Liang, K. Patterns of HIV and SARS-CoV-2 co-infection in Wuhan, China. J. Int. AIDS Soc. 2020, 23, e25568. [CrossRef]

55. Haddad, S.; Tayyar, R.; Risch, L.; Churchill, G.; Fares, E.; Choe, M.; Montemuro, P. Encephalopathy and seizure activity in a COVID-19 well controlled HIV patient. IDCases 2020, 21, e00814.

56. Hadi, Y.B.; Naqvi, S.F.; Kupec, J.T.; Sarwari, A.R. Characteristics and outcomes of COVID-19 in patients with HIV: A multicentre research network study. AIDS 2020, 34, 3-8.

57. Haerter, G.; Spinner, C.D.; Roider, J.; Bickel, M.; Krznaric, I.; Grunwald, S.; Schabaz, F.; Gillor, D.; Postel, N.; Mueller, M.C. COVID-19 in people living with human immunodeficiency virus: A case series of 33 patients. Infection 2020, 48, 681-686.

58. Ho, H.-e.; Peluso, M.J.; Margus, C.; Matias Lopes, J.P.; He, C.; Gaisa, M.M.; Osorio, G.; Aberg, J.A.; Mullen, M.P. Clinical outcomes and immunologic characteristics of Covid-19 in people with HIV. J. Infect. Dis. 2020, 223, 403-408.

59. Hu, Y.; Ma, J.; Huang, H.; Vermund, S.H. Coinfection With HIV and SARS-CoV-2 in Wuhan, China: A 12-Person Case Series. J. Acquir. Immune Defic. Syndr. 2020, 85, 1-5. [CrossRef]

60. Huang, J.; Xie, N.; Hu, X.; Yan, H.; Ding, J.; Liu, P.; Ma, H.; Ruan, L.; Li, G.; He, N. Epidemiological, virological and serological features of COVID-19 cases in people living with HIV in Wuhan City: A population-based cohort study. Clin. Infect. Dis. 2020. [CrossRef]

61. Iordanou, S.; Koukios, D.; Matsentidou, C.T.; Markoulaki, D.; Raftopoulos, V. Severe SARS-CoV-2 pneumonia in a 58-year-old patient with HIV: A clinical case report from the Republic of Cyprus. J. Med Virol. 2020, 92, 2361-2365.

62. Isernia, V.; Julia, Z.; Le Gac, S.; Bachelard, A.; Landman, R.; Lariven, S.; Joly, V.; Deconinck, L.; Rioux, C.; Lescure, X. SARS-COV2 infection in 30 HIV-infected patients followed-up in a French University Hospital. Int. J. Infect. Dis. 2020, 101, 49-51.

63. Karmen-Tuohy, S.; Carlucci, P.M.; Zervou, F.N.; Zacharioudakis, I.M.; Rebick, G.; Klein, E.; Reich, J.; Jones, S.; Rahimian, J. Outcomes Among HIV-Positive Patients Hospitalized With COVID-19. J. Acquir. Immune Defic. Syndr. 2020, 85, 6-10.

64. Khaba, M.C.; Ngale, T.C.; Madala, N. COVID-19 in an HIV-infected patient. Lessons learned from an autopsy case. Int. J. Infect. Dis. 2020, 101, 243-246.

65. Kim, J.-Y.; Kim, J.M.; Peck, K.R. The First Case of an HIV Patient Diagnosed with COVID-19 in Korea. J. Korean Med. Sci. 2020, 35, e358.

66. Kumar, R.N.; Tanna, S.D.; Shetty, A.A.; Stosor, V. COVID-19 in an HIV-positive Kidney Transplant Recipient. Transpl. Infect. Dis. 2020, 22, e13338.

67. Li, W.; Ma, Q.; Wang, X.; Tang, M.; Lin, J.; Xiao, B. The characteristics of two patients coinfected with SARS-CoV-2 and HIV in Wuhan, China. J. Med Virol. 2020, 93, 85-88.

68. Liu, J.; Zeng, W.; Cao, Y.; Cui, Y.; Li, Y.; Yao, S.; Alwalid, O.; Yang, F.; Fan, Y.; Shi, H. Effect of a previous history of antiretroviral treatment on the clinical picture of patients with co-infection of SARS-CoV-2 and HIV: A preliminary study. Int. J. Infect. Dis. 2020, 100, 141-148.

69. Madge, S.; Barber, T.J.; Hunter, A.; Bhagani, S.; Lipman, M.; Burns, F. Descriptive account of 18 adults with known HIV infection hospitalised with SARS-CoV-2 infection. Sex. Transm. Infect. 2020. [CrossRef] 
70. Mang, S.; Kaddu-Mulindwa, D.; Metz, C.; Becker, A.; Seiler, F.; Smola, S.; Maßmann, A.; Becker, S.L.; Papan, C.; Bals, R. Pneumocystis jirovecii pneumonia and SARS-CoV-2 co-infection in newly diagnosed HIV-1 infection. Clin. Infect. Dis. Off. Publ. Infect. Dis. Soc. Am. 2020. [CrossRef]

71. Marimuthu, J.; Kumar, B.S.; Gandhi P, A. HIV and SARS CoV-2 coinfection: A retrospective, record-based, case series from South India. J. Med Virol. 2020, 93, 163-165.

72. Meyerowitz, E.A.; Kim, A.Y.; Ard, K.L.; Basgoz, N.; Chu, J.T.; Hurtado, R.M.; Lee, C.K.; He, W.; Minukas, T.; Nelson, S.; et al. Disproportionate burden of coronavirus disease 2019 among racial minorities and those in congregate settings among a large cohort of people with HIV. AIDS 2020, 34, 1781-1787.

73. Miyashita, H.; Kuno, T. Prognosis of coronavirus disease 2019 (COVID-19) in patients with HIV infection in New York City. HIV Med. 2020. [CrossRef]

74. Modi, A.R.; Koval, C.E.; Taege, A.J.; Modaresi Esfeh, J.; Eghtesad, B.; Menon, K.N.; Quintini, C.; Miller, C. Coronavirus Disease 2019 in an Orthotopic Liver Transplant Recipient Living with Human Immunodeficiency Virus. Transpl. Infect. Dis. 2020, 22, e13351.

75. Molina-Iturritza, E.; San-José-Muñiz, I.; Ganchegui-Aguirre, M.; Balerdi-Sarasola, L.; Ortiz-de-Zárate-Ibarra, Z.; GainzarainArana, J.C.; Portu-Zapirain, J. Coronavirus disease 2019 in patients with HIV in the province of Araba, Basque Country, Spain. AIDS 2020, 34, 1696-1697. [CrossRef]

76. Mondi, A.; Cimini, E.; Colavita, F.; Cicalini, S.; Pinnetti, C.; Matusali, G.; Casetti, R.; Maeurer, M.; Vergori, A.; Mazzotta, V. COVID-19 in people living with HIV: Clinical implications of dynamics of immune response to SARS-CoV-2. J. Med Virol. 2020, 93, 1796-1804.

77. Nakamoto, T.; Kutsuna, S.; Yanagawa, Y.; Kanda, K.; Okuhama, A.; Akiyama, Y.; Miyazato, Y.; Ide, S.; Nakamura, K.; Yamamoto, K. A case of SARS-CoV-2 infection in an untreated HIV patient in Tokyo, Japan. J. Med Virol. 2020, 93, 40-42.

78. Okoh, A.K.; Bishburg, E.; Grinberg, S.; Nagarakanti, S. COVID-19 Pneumonia in Patients With HIV: A Case Series. J. Acquir. Immune Defic. Syndr. 2020, 85, e4-e5. [CrossRef]

79. Parker, A.; Shaw, J.; Karamchand, S.; Lahri, S.; Schrueder, N.; Chothia, M.Y.; Mowlana, A.; Lalla, U.; Allwood, B.W.; Koegelenberg, C.F.N.; et al. HIV and SARS-CoV-2 co-infection: The diagnostic challenges of dual pandemics. S. Afr. Med. J. Suid Afrik. Tydskr. Geneeskd. 2020, 110, 473-475. [CrossRef]

80. Parker, A.; Koegelenberg, C.F.; Moolla, M.S.; Louw, E.H.; Mowlana, A.; Nortjé, A.; Ahmed, R.; Brittain, N.; Lalla, U.; Allwood, B.W.; et al. High HIV prevalence in an early cohort of hospital admissions with COVID-19 in Cape Town, South Africa. SAMJ S. Afr. Med. J. 2020, 110, 982.

81. Patel, R.H.; Pella, P.M. COVID-19 in a patient with HIV infection. J. Med. Virol. 2020, 92, 2356-2357.

82. Przydzial, P.; Tchomobe, G.; Amin, K.; Engell, C.A.; Okoh, A.K. COVID-19 crossing paths with AIDS in the homeless. J. Med. Virol. 2020, 93, 155-157.

83. Qasim, A.; Mansour, M.; Kousa, O.; Awad, D.; Abuhazeem, B.; Millner, P.; Velagapudi, M. A case of coronavirus disease 2019 in acquired immunodeficiency syndrome patient: A case report and review of the literature. Intractable Rare Dis. Res. 2020, 9 , 256-259.

84. Ridgway, J.P.; Farley, B.; Benoit, J.-L.; Frohne, C.; Hazra, A.; Pettit, N.; Pho, M.; Pursell, K.; Saltzman, J.; Schmitt, J.; et al. A Case Series of Five People Living with HIV Hospitalized with COVID-19 in Chicago, Illinois. AIDS Patient Care STDs 2020, 34, 331-335. [CrossRef]

85. Riva, A.; Conti, F.; Bernacchia, D.; Pezzati, L.; Sollima, S.; Merli, S.; Siano, M.; Lupo, A.; Rusconi, S.; Cattaneo, D.; et al. Darunavir does not prevent SARS-CoV-2 infection in HIV patients. Pharmacol. Res. 2020, 157, 104826. [CrossRef]

86. Rivas, N.; Espinoza, M.; Loban, A.; Luque, O.; Jurado, J.; Henry-Hurtado, N.; Goodridge, A. Case Report: COVID-19 Recovery from Triple Infection with Mycobacterium tuberculosis, HIV, and SARS-CoV-2. Am. J. Trop. Med. Hyg. 2020, 103, 1597-1599.

87. Ruan, L.; Zhang, Y.; Luo, Y.; Yu, X.; Zeng, Y.; Peng, H.; Han, L.; Chen, L.; Roy, S.; Cheng, Q.; et al. Clinical features and outcomes of four HIV patients with COVID-19 in Wuhan, China. J. Med. Virol. 2020, 93, 133-136.

88. Sasset, L.; Di Meco, E.; Cavinato, S.; Cattelan, A.M. Coinfection of severe acute respiratory syndrome coronavirus 2 and HIV in a teaching hospital: Still much to learn. AIDS 2020, 34, 1694-1696. [CrossRef]

89. Shalev, N.; Scherer, M.; LaSota, E.D.; Antoniou, P.; Yin, M.T.; Zucker, J.; Sobieszczyk, M.E. Clinical Characteristics and Outcomes in People Living With Human Immunodeficiency Virus Hospitalized for Coronavirus Disease 2019. Clin. Infect. Dis. 2020, 71, 2294-2297.

90. Shekhar, R.; Barton, A.; Sheikh, A.B.; Upadhyay, S.; Salas, N.M. Coronavirus Disease of 2019 in Patients With Well-Controlled Human Immunodeficiency Virus on Antiretroviral Therapy. J. Acquir. Immune Defic. Syndr. 2020, 85, e1-e4. [CrossRef]

91. Sigel, K.; Swartz, T.; Golden, E.; Paranjpe, I.; Somani, S.; Richter, F.; De Freitas, J.K.; Miotto, R.; Zhao, S.; Polak, P. COVID-19 and people with HIV infection: Outcomes for hospitalized patients in New York City. Clin. Infect. Dis. 2020, 71, $2933-2938$.

92. Stoeckle, K.; Johnston, C.D.; Jannat-Khah, D.P.; Williams, S.C.; Ellman, T.M.; Vogler, M.A.; Gulick, R.M.; Glesby, M.J.; Choi, J.J. COVID-19 in Hospitalized Adults With HIV. Open Forum Infect. Dis. 2020, 7, ofaa327.

93. Su, J.; Shen, X.; Ni, Q.; Zhao, H.; Cai, J.; Zhu, B.; Wu, W.; Lang, G.; Xu, K.; Sheng, J. Infection of severe acute respiratory syndrome coronavirus 2 in a patient with AIDS. AIDS 2020,34, 1575-1576. [CrossRef]

94. Sun, L.J.; Wong, S.X.L.; Gollamudi, S. A Case of HIV and SARS-CoV-2 Co-infection in Singapore. J. Acquir. Immune Defic. Syndr. 2020, 84, e23-e24. [CrossRef] 
95. Suwanwongse, K.; Shabarek, N. Clinical features and outcome of HIV/SARS-CoV-2 co-infected patients in the Bronx, New York City. J. Med. Virol. 2020. [CrossRef]

96. Suwanwongse, K.; Shabarek, N. Variation in mortality of HIV/SARS-CoV-2 coinfected patients in the Bronx, New York City. J. Med. Virol. 2020, 93, 603-605.

97. Toombs, J.M.; Van den Abbeele, K.; Democratis, J.; Merricks, R.; Mandal, A.K.; Missouris, C.G. COVID-19 in three people living with HIV in the United Kingdom. J. Med. Virol. 2020, 93, 107-109. [CrossRef]

98. Wang, M.; Luo, L.; Bu, H.; Xia, H. Case Report: One Case of Coronavirus Desease 2019 (COVID-19) in Patient Co-nfected by HIV With a Low CD4+ T Cell Count. Int. J. Infect. Dis. 2020, 96, 148-150.

99. Wu, M.; Luo, L.; Bu, H.; Xia, H. Case report: One case of coronavirus disease 2019 (COVID-19) in a patient co-infected by HIV with a normal CD4(+) T cell count. AIDS Res. Ther. 2020, 17, 020-00301.

100. Wu, Q.; Chen, T.; Zhang, H. Recovery from COVID-19 in two patients with coexisted HIV infection. J. Med. Virol. 2020, 92, 2325-2327.

101. Yamamoto, S.; Saito, M.; Nagai, E.; Toriuchi, K.; Nagai, H.; Yotsuyanagi, H.; Nakagama, Y.; Kido, Y.; Adachi, E. Antibody Response to SARS-CoV-2 in people living with HIV. J. Microbiol. Immunol. Infect. Wei Mian Yu Gan Ran Za Zhi 2020, 54, 144-146.

102. Zhang, J.-C.; Yu, X.-H.; Ding, X.-H.; Ma, H.-Y.; Cai, X.-Q.; Kang, S.-C.; Xiang, D.-W. New HIV diagnoses in patients with COVID-19: Two case reports and a brief literature review. BMC Infect. Dis. 2020, 20, 1-10.

103. Zhao, J.; Liao, X.; Wang, H.; Wei, L.; Xing, M.; Liu, L. Early Virus Clearance and Delayed Antibody Response in a Case of Coronavirus Disease 2019 (COVID-19) With a History of Coinfection With Human Immunodeficiency Virus Type 1 and Hepatitis C Virus. Clin. Infect. Dis. 2020, 71, 2233-2235.

104. Zhu, F.; Cao, Y.; Xu, S.; Zhou, M. Co-infection of SARS-CoV-2 and HIV in a patient in Wuhan city, China. J. Med. Virol. 2020, 92, 529-530. [CrossRef]

105. WHO. Guidelines for HIV Diagnosis and Monitoring of Antiretroviral Therapy, Rev. 2; WHO Regional Office for South-East Asia: New Delhi, India, 2009.

106. Akiyama, S.; Hamdeh, S.; Micic, D.; Sakuraba, A. Prevalence and clinical outcomes of COVID-19 in patients with autoimmune diseases: A systematic review and meta-analysis. Ann. Rheum. Dis. 2020, 80, 384-391.

107. Hu, Y.; Sun, J.; Dai, Z.; Deng, H.; Li, X.; Huang, Q.; Wu, Y.; Sun, L.; Xu, Y. Prevalence and severity of corona virus disease 2019 (COVID-19): A systematic review and meta-analysis. J. Clin. Virol. 2020, 127, 104371.

108. Baradaran, A.; Ebrahimzadeh, M.H.; Baradaran, A.; Kachooei, A.R. Prevalence of comorbidities in COVID-19 patients: A systematic review and meta-analysis. Arch. Bone Joint Surg. 2020, 8, 247.

109. Bajgain, K.T.; Badal, S.; Bajgain, B.B.; Santana, M.J. Prevalence of comorbidities among individuals with COVID-19: A rapid review of current literature. Am. J. Infect. Control. 2020, 49, 238-246.

110. Gold, M.S.; Sehayek, D.; Gabrielli, S.; Zhang, X.; McCusker, C.; Ben-Shoshan, M. COVID-19 and comorbidities: A systematic review and meta-analysis. Postgrad. Med. 2020, 132, 749-755.

111. Qiu, P.; Zhou, Y.; Wang, F.; Wang, H.; Zhang, M.; Pan, X.; Zhao, Q.; Liu, J. Clinical characteristics, laboratory outcome characteristics, comorbidities, and complications of related COVID-19 deceased: A systematic review and meta-analysis. Aging Clin. Exp. Res. 2020, 32, 1-10.

112. Pranata, R.; Lim, M.A.; Huang, I.; Raharjo, S.B.; Lukito, A.A. Hypertension is associated with increased mortality and severity of disease in COVID-19 pneumonia: A systematic review, meta-analysis and meta-regression. J. Renin Angiotensin Aldosterone Syst. 2020, 21, 1470320320926899.

113. Mills, K.T.; Stefanescu, A.; He, J. The global epidemiology of hypertension. Nat. Rev. Nephrol. 2020, 16, $223-237$.

114. Schiffrin, E.L.; Flack, J.M.; Ito, S.; Muntner, P.; Webb, R.C. Hypertension and COVID-19. Am. J. Hypertens. 2020, 33, 373-374. [CrossRef]

115. Fang, L.; Karakiulakis, G.; Roth, M. Are patients with hypertension and diabetes mellitus at increased risk for COVID-19 infection? Lancet Respir. Med. 2020, 8, e21.

116. Furuhashi, M.; Moniwa, N.; Mita, T.; Fuseya, T.; Ishimura, S.; Ohno, K.; Shibata, S.; Tanaka, M.; Watanabe, Y.; Akasaka, H. Urinary angiotensin-converting enzyme 2 in hypertensive patients may be increased by olmesartan, an angiotensin II receptor blocker. Am. J. Hypertens. 2015, 28, 15-21.

117. Ferrario, C.M.; Jessup, J.; Chappell, M.C.; Averill, D.B.; Brosnihan, K.B.; Tallant, E.A.; Diz, D.I.; Gallagher, P.E. Effect of angiotensinconverting enzyme inhibition and angiotensin II receptor blockers on cardiac angiotensin-converting enzyme 2. Circulation 2005, 111, 2605-2610.

118. Vaduganathan, M.; Vardeny, O.; Michel, T.; McMurray, J.J.; Pfeffer, M.A.; Solomon, S.D. Renin-angiotensin-aldosterone system inhibitors in patients with Covid-19. N. Engl. J. Med. 2020, 382, 1653-1659.

119. Shi, H.; Han, X.; Jiang, N.; Cao, Y.; Alwalid, O.; Gu, J.; Fan, Y.; Zheng, C. Radiological findings from 81 patients with COVID-19 pneumonia in Wuhan, China: A descriptive study. Lancet Infect. Dis. 2020, 20, 425-434.

120. Pan, F.; Ye, T.; Sun, P.; Gui, S.; Liang, B.; Li, L.; Zheng, D.; Wang, J.; Hesketh, R.L.; Yang, L. Time course of lung changes on chest CT during recovery from 2019 novel coronavirus (COVID-19) pneumonia. Radiology 2020. [CrossRef]

121. Awulachew, E.; Diriba, K.; Anja, A.; Getu, E.; Belayneh, F. Computed Tomography (CT) Imaging features of patients with COVID-19: Systematic review and meta-analysis. Radiol. Res. Pr. 2020, 2020, 1023506. 
122. Cao, Y.; Liu, X.; Xiong, L.; Cai, K. Imaging and clinical features of patients with 2019 novel coronavirus SARS-CoV-2: A systematic review and meta-analysis. J. Med. Virol. 2020, 92, 1449-1459.

123. Ng, M.-Y.; Lee, E.Y.; Yang, J.; Yang, F.; Li, X.; Wang, H.; Lui, M.M.-s.; Lo, C.S.-Y.; Leung, B.; Khong, P.-L. Imaging profile of the COVID-19 infection: Radiologic findings and literature review. Radiol. Cardiothorac. Imaging 2020, 2, e200034.

124. Salehi, S.; Abedi, A.; Balakrishnan, S.; Gholamrezanezhad, A. Coronavirus disease 2019 (COVID-19): A systematic review of imaging findings in 919 patients. Am. J. Roentgenol. 2020, 215, 87-93.

125. Abdullahi, A.; Candan, S.A.; Abba, M.A.; Bello, A.H.; Alshehri, M.A.; Afamefuna Victor, E.; Umar, N.A.; Kundakci, B. Neurological and musculoskeletal features of COVID-19: A systematic review and meta-analysis. Front. Neurol. 2020, 11, 687.

126. Chua, T.H.; Xu, Z.; King, N.K.K. Neurological manifestations in COVID-19: A systematic review and meta-analysis. Brain Inj. 2020, 34, 1549-1568.

127. Collantes, M.E.V.; Espiritu, A.I.; Sy, M.C.C.; Anlacan, V.M.M.; Jamora, R.D.G. Neurological manifestations in COVID-19 infection: A systematic review and meta-analysis. Can. J. Neurol. Sci. J. Can. Sci. Neurol. 2021, 48, 66-76.

128. Wu, Y.; Xu, X.; Chen, Z.; Duan, J.; Hashimoto, K.; Yang, L.; Liu, C.; Yang, C. Nervous system involvement after infection with COVID-19 and other coronaviruses. Brain Behav. Immun. 2020, 87, 18-22.

129. Xu, H.; Zhong, L.; Deng, J.; Peng, J.; Dan, H.; Zeng, X.; Li, T.; Chen, Q. High expression of ACE2 receptor of 2019-nCoV on the epithelial cells of oral mucosa. Int. J. Oral Sci. 2020, 12, 1-5.

130. Li, G.; He, X.; Zhang, L.; Ran, Q.; Wang, J.; Xiong, A.; Wu, D.; Chen, F.; Sun, J.; Chang, C. Assessing ACE2 expression patterns in lung tissues in the pathogenesis of COVID-19. J. Autoimmun. 2020, 112, 102463.

131. Baig, A.M.; Khaleeq, A.; Ali, U.; Syeda, H. Evidence of the COVID-19 virus targeting the CNS: Tissue distribution, host-virus interaction, and proposed neurotropic mechanisms. ACS Chem. Neurosci. 2020, 11, 995-998.

132. Chilvers, M.; McKean, M.; Rutman, A.; Myint, B.; Silverman, M.; O'Callaghan, C. The effects of coronavirus on human nasal ciliated respiratory epithelium. Eur. Respir. J. 2001, 18, 965-970.

133. Chen, X.; Laurent, S.; Onur, O.A.; Kleineberg, N.N.; Fink, G.R.; Schweitzer, F.; Warnke, C. A systematic review of neurological symptoms and complications of COVID-19. J. Neurol. 2020, 268, 1-11.

134. Ghahramani, S.; Tabrizi, R.; Lankarani, K.B.; Kashani, S.M.A.; Rezaei, S.; Zeidi, N.; Akbari, M.; Heydari, S.T.; Akbari, H.; Nowrouzi-Sohrabi, P. Laboratory features of severe vs. non-severe COVID-19 patients in Asian populations: A systematic review and meta-analysis. Eur. J. Med. Res. 2020, 25, 1-10.

135. Martins-Chaves, R.R.; Gomes, C.C.; Gomez, R.S. Immunocompromised patients and coronavirus disease 2019: A review and recommendations for dental health care. Braz. Oral Res. 2020, 34, e048.

136. WHO. COVID-19 Significantly Impacts Health Services for Noncommunicable Diseases. Available online: https://www.who.int/ news/item/01-06-2020-covid-19-significantly-impacts-health-services-for-noncommunicable-diseases (accessed on 10 March 2020).

137. Lagat, H.; Sharma, M.; Kariithi, E.; Otieno, G.; Katz, D.; Masyuko, S.; Mugambi, M.; Wamuti, B.; Weiner, B.; Farquhar, C. Impact of the COVID-19 pandemic on HIV testing and assisted partner notification services, Western Kenya. AIDS Behav. 2020, 24, 3010-3013.

138. Hogan, A.B.; Jewell, B.L.; Sherrard-Smith, E.; Vesga, J.F.; Watson, O.J.; Whittaker, C.; Hamlet, A.; Smith, J.A.; Winskill, P.; Verity, R. Potential impact of the COVID-19 pandemic on HIV, tuberculosis, and malaria in low-income and middle-income countries: A modelling study. Lancet Glob. Health 2020, 8, e1132-e1141.

139. Kowalska, J.D.; Skrzat-Klapaczyńska, A.; Bursa, D.; Balayan, T.; Begovac, J.; Chkhartishvili, N.; Gokengin, D.; Harxhi, A.; Jilich, D.; Jevtovic, D. HIV care in times of the COVID-19 crisis-Where are we now in Central and Eastern Europe? Int. J. Infect. Dis. 2020, 96, 311-314.

140. Jiang, H.; Zhou, Y.; Tang, W. Maintaining HIV care during the COVID-19 pandemic. Lancet HIV 2020, 7, e308-e309.

141. Chenneville, T.; Gabbidon, K.; Hanson, P.; Holyfield, C. The impact of COVID-19 on HIV treatment and research: A call to action. Int. J. Environ. Res. Public Health 2020, 17, 4548.

142. Wakoli, A.K.; Ememwa, K.U. Focus on Multi-month dispensing of Antiretroviral Therapy among HIV-infected patients in Kenya during the COVID-19 lockdown period. Kenyan J. Nurs. Midwifery 2020, 5, 82-88.

143. Oliver, S.E.; Gargano, J.W.; Marin, M.; Wallace, M.; Curran, K.G.; Chamberland, M.; McClung, N.; Campos-Outcalt, D.; Morgan, R.L.; Mbaeyi, S. The Advisory Committee on Immunization Practices' Interim Recommendation for Use of Pfizer-BioNTech COVID-19 Vaccine-United States, December 2020. Morb. Mortal. Wkly. Rep. 2020, 69, 1922.

144. Oliver, S.E. The Advisory Committee on Immunization Practices' Interim Recommendation for Use of Moderna COVID-19 Vaccine-United States, December 2020. Morb. Mortal. Wkly. Rep. 2020, 69, 1653-1656.

145. Voysey, M.; Clemens, S.A.C.; Madhi, S.A.; Weckx, L.Y.; Folegatti, P.M.; Aley, P.K.; Angus, B.; Baillie, V.L.; Barnabas, S.L.; Bhorat, Q.E. Single-dose administration and the influence of the timing of the booster dose on immunogenicity and efficacy of ChAdOx1 nCoV-19 (AZD1222) vaccine: A pooled analysis of four randomised trials. Lancet 2021, 397, 881-891.

146. Sadoff, J.; Le Gars, M.; Shukarev, G.; Heerwegh, D.; Truyers, C.; de Groot, A.M.; Stoop, J.; Tete, S.; Van Damme, W.; Leroux-Roels, I. Interim Results of a Phase 1-2a Trial of Ad26. COV2. S Covid-19 Vaccine. N. Engl. J. Med. 2021. [CrossRef]

147. Polack, F.P.; Thomas, S.J.; Kitchin, N.; Absalon, J.; Gurtman, A.; Lockhart, S.; Perez, J.L.; Pérez Marc, G.; Moreira, E.D.; Zerbini, C. Safety and efficacy of the BNT162b2 mRNA Covid-19 vaccine. N. Engl. J. Med. 2020, 383, 2603-2615.

148. Baden, L.R.; El Sahly, H.M.; Essink, B.; Kotloff, K.; Frey, S.; Novak, R.; Diemert, D.; Spector, S.A.; Rouphael, N.; Creech, C.B. Efficacy and safety of the mRNA-1273 SARS-CoV-2 vaccine. N. Engl. J. Med. 2021, 384, 403-416. 